\title{
Regularity results for nonlinear Young equations and applications
}

\author{
DAvide Addona, Luca Lorenzi And Gianmario Tessitore@
}

Abstract. In this paper we provide sufficient conditions which ensure that the nonlinear equation $\mathrm{d} y(t)=$ $A y(t) \mathrm{d} t+\sigma(y(t)) \mathrm{d} x(t), t \in(0, T]$, with $y(0)=\psi$ and $A$ being an unbounded operator, admits a unique mild solution such that $y(t) \in D(A)$ for any $t \in(0, T]$, and we compute the blow-up rate of the norm of $y(t)$ as $t \rightarrow 0^{+}$. We stress that the regularity of $y$ is independent of the smoothness of the initial datum $\psi$, which in general does not belong to $D(A)$. As a consequence we get an integral representation of the mild solution $y$ which allows us to prove a chain rule formula for smooth functions of $y$.

\section{Introduction}

The Young integral has been introduced in [15], where the author defines extension of the Riemann-Stieltjes integral $\int f d g$ when neither $f$ nor $g$ have finite total variation. In particular in [15] it is shown that, if $f$ and $g$ are continuous functions such that $f$ has finite $p$-variation and $g$ has finite $q$-variation, with $p, q>0$ and $p^{-1}+q^{-1}>1$, then the Stieltjes integral $\int f d g$ is well-defined as a limit of Riemann sums. This was the starting point of the crucial extension to rough paths integration. Indeed, in [13] the author proves that it is possible to define the integral $\int f \mathrm{~d} x$ also in the case when $f$ has finite $p$-variation and $x$ has finite $q$-variation with $p, q>0$ and $p^{-1}+q^{-1}<1$. In this case, additional information on the function $x$ is needed, which would play the role of iterated integrals for regular paths.

An alternative formulation of the integration over rough paths is provided in [6], where the author considers Hölder-like (semi)norms instead of $p$-variation norms. Namely, if $f$ is $\alpha$-Hölder continuous and $g$ is $\eta$-Hölder continuous with $\alpha+\eta>1$ then the Young integral is well defined as the unique solution to an algebraic problem. Recently, a more general theory of rough integration, when $\alpha+\eta \leq 1$, has been introduced in [5].

Here, we consider only Young integrals and focus on the spatial regularity of solutions to infinite dimensional evolution equations leaving aside the enormous amount of results connected to the rough paths case culminating in the breakthrough on singular

Mathematics Subject Classification: Primary: 35R60; Secondary: 60H05, 60H15, 47D06

Keywords: Nonlinear Young equations, Mild solutions and their smoothness, Integral representation formula, Semigroups of bounded operators, Invariance property. 
SPDEs (see, e.g., [7]). Namely, we consider the nonlinear evolution equation

$$
\left\{\begin{array}{l}
\mathrm{d} y(t)=A y(t) \mathrm{d} t+\sigma(y(t)) \mathrm{d} x(t), \quad t \in(0, T] \\
y(0)=\psi
\end{array}\right.
$$

where $A$ is the infinitesimal generator of a semigroup defined on a Banach space $X$ with suitable regularizing properties and $x$ is a $\eta$-Hölder continuous function with $\eta>1 / 2$. Ordinary differential equations (in finite dimensional spaces) driven by an irregular path of Hölder regularity greater than $1 / 2$ have been understood in full details since [16] (see also [10]). On the other hand, the infinite dimensional case was treated in [8] and then developed in [9] and [3], see also [14] for earlier results in the context of stochastic partial differential equations driven by an infinite dimensional fractional Brownian motion of Hurst parameter $H>1 / 2$.

In [3], problem (1.1) is formulated in a mild form

$$
y(t)=S(t) y(0)+\int_{0}^{t} S(t-r)(\sigma(y(r))) \mathrm{d} x(r), \quad t \in[0, T],
$$

where $(S(t))_{t \geq 0}$ is the analytic semigroup generated by the sectorial operator $A$, and the authors exploit the regularizing properties of $S$ to show that, if the initial datum $\psi$ is smooth enough (i.e., if it belongs to a suitable domain of the fractional powers $\left.(-A)^{\alpha}\right)$, then Eq. (1.1) admits a unique mild solution with the same spatial regularity as the initial datum. The key technical point in [3] is to prove that the convolution

$$
\int_{0}^{t} S(t-s) f(s) \mathrm{d} x(s)
$$

is well defined if $f$ takes values in $D\left((-A)^{\alpha}\right)$ and belongs to a Hölder-type function space. To be more precise, the authors require that $f:[0, T] \rightarrow D\left((-A)^{\alpha}\right)$ satisfies the condition

$$
\sup _{s<t, s, t \in[0, T]} \frac{\|f(t)-f(s)-(S(t-s)-I) f(s)\|_{D\left((-A)^{\alpha}\right)}}{(t-s)^{\beta}}<+\infty .
$$

This is one of the main difference with respect to the finite dimensional case, where the condition on the function $f$ reads in terms of classical Hölder norms. Once that convolution (1.2) is well-defined, the smoothness of the initial datum $\psi$ and suitable estimates on (1.2) allow the authors to solve the mild reformulation of Eq. (1.1) by a fixed point argument in the same Hölder-type function space introduced above.

Our point in the present paper is that, if one looks a bit more closely to the tradeoff between Hölderianity in time and regularity in space of the convolution (1.2), one discovers that an extra regularity in space can be extracted by estimates, see Lemma 2.2. This allows us to show that the mild solution to Eq. (1.1), which in our situation is driven by a finite dimensional noise, is more regular than the initial datum (that nevertheless has to enjoy the same regularity assumptions as in [3]). Namely, $y(t)$ belongs to $D(A)$ for any $t \in(0, T]$ (see Theorem 3.1). 
It is also worth mentioning that, when $A$ is an unbounded operator, the mild formulation of Eq. (1.1) is the most suitable to prove existence and uniqueness of a solution since it allows to apply a fixed point argument in spaces of functions with a low degree of smoothness. On the other hand this formulation is too weak in several applications where an integral formulation of the equation helps a lot. Here, having proved that the mild solution $y$ takes values in $D(A)$, we are in a position to show that $y$ admits an integral representation as well, i.e., it satisfies the equation:

$$
y(t)=\psi+\int_{0}^{t} A y(s) \mathrm{d} s+\int_{0}^{t} \sigma(y(s)) \mathrm{d} x(s), \quad t \in[0, T] .
$$

Moreover, starting from the above relation, we can also obtain a chain rule; in other words we show that we can differentiate with respect to time regular enough functions of the solution to Eq. (1.1). Finally, as an example of possible applications of the chain rule, we propose (in Hilbertian setting, see Proposition 5.1) a necessary conditions for the invariance of Hyperplanes under the action of solutions of equations driven by irregular paths. In the case of an ordinary differential equation with a rough path, this problem is addressed in [2], when the state space is finite dimensional and no unbounded operators are involved in the equation. The problem of the invariance of a convex set with respect to a general infinite dimensional evolution equation driven by a rough trajectory is still unexplored (see [1] and the references therein for corresponding results in the case of classical evolution equations).

Summarizing, this paper can been described as a first step towards a systematic study, by the classical tools of semigroup theory, of smoothing properties of the mild solution to (1.1). We plan to go further in the analysis, first weakening the smoothness assumptions on $\psi$ and, then, developing results analogous to those in this paper for equations driven by more irregular noises as in the case of rough paths.

The paper is structured as follows. In Sect. 2, we introduce the function spaces that we use and we recall some results taken from [6,9], slightly generalizing some of those results. In Sect. 3, we prove the existence and uniqueness of a mild solution to the nonlinear Young equation (1.1) when $\psi$ belongs to a suitable space $X_{\alpha} \subset X$ (which will be defined later), $x$ is $\eta$-Hölder continuous for some $\eta \in(1 / 2,1)$ and $\alpha+\eta>1$. We show that this solution takes values in $D(A)$ and estimate the blow-up rate of its $X_{1+\mu}$-norm as $t$ tends to $0^{+}$, when $\mu \in[0, \eta+\alpha-1)$ (see Theorem 3.1). The smoothness of the mild solution strongly relies on the smoothing effect of the semigroup associated with operator $A$. In general, when $A$ is the infinitesimal generator of a strongly continuous semigroup, such smoothing properties are not satisfied by the associated semigroup. Nevertheless, we still can prove the existence and uniqueness of the mild equation to Eq. (1.1) by a suitable choice of the spaces $X_{\alpha}$. Based on Theorem 3.1, in Sect. 4 we prove that the mild solution to (1.1) can be written in an integral form, which is used in Sect. 5 to prove the chain rule. By a simple example, we show how the availability both of a solution, which takes values in $D(A)$, and of a chain rule, can be exploited to tackle the problem of the invariance of convex sets, when an unbounded operator $A$ is involved. Finally, in Sect. 6 we provide two 
examples, one on the space of continuous functions and one on an $L^{p}$ state space, to illustrate our results.

Notation. We denote by $[a, b]_{<}^{2}$ the set $\left\{(s, t) \in \mathbb{R}^{2}: a \leq s<t \leq b\right\}$. Further, we denote by $\mathscr{L}\left(X_{\alpha}, X_{\gamma}\right)$ the space of linear bounded operators from $X_{\alpha}$ into $X_{\gamma}$, for each $\alpha, \gamma \geq 0$. For every $A \subseteq \mathbb{R}, C(A ; X)$ denotes the usual space of continuous functions from $A$ into $X$ endowed with the sup-norm. The subscript " $b$ " stands for bounded. Finally, for every $\alpha \in(0,1), C^{\alpha}(A ; X)$ denotes the subset of $C_{b}(A ; X)$ consisting of $\alpha$ Hölder continuous functions. It is endowed with the norm $\|f\|_{C^{\alpha}(A ; X)}=\|f\|_{C_{b}(A ; X)}+$ $[f]_{C^{\alpha}(A ; X)}$, where $[f]_{C^{\alpha}(A ; X)}=\sup _{\substack{s, t \in A \\ s \neq t}} \frac{\|f(t)-f(s)\|_{X}}{|t-s|^{\alpha}}$. When $X=\mathbb{R}$, we simply write $C^{\alpha}(A)$.

\section{The abstract Young equation}

\subsection{Function spaces and preliminary results}

Throughout the paper, $X$ denotes a Banach space and $A: D(A) \subseteq X \rightarrow X$ is a linear operator which generates a semigroup $(S(t))_{t \geq 0}$. We further assume the following set of assumptions.

Hypothesis 2.1. (i) For every $\alpha \in[0,2)$, there exists a space $X_{\alpha}$ (with the convention that $X_{0}=X$ and $X_{1}=D(A)$ ) such that if $\beta \leq \alpha$ then $X_{\alpha}$ is continuously embedded into $X_{\beta}$. We denote by $K_{\alpha, \beta}$ a positive constant such that $|x|_{\beta} \leq K_{\alpha, \beta}|x|_{\alpha}$ for every $x \in X_{\alpha}$;

(ii) for every $\zeta, \alpha, \gamma \in[0,2), \zeta \leq \alpha$, and $\mu, v \in(0,1]$ with $\mu>v$ there exist positive constants $M_{\zeta, \alpha, T}$, and $C_{\mu, \nu, T}$, which depend on $T$, such that

$$
\left\{\begin{array}{l}
\left(\text { a) }\|S(t)\|_{\mathscr{L}\left(X_{\zeta}, X_{\alpha}\right)} \leq M_{\zeta, \alpha, T} t^{-\alpha+\zeta}\right. \\
(b)\|S(t)-I\|_{\mathscr{L}\left(X_{\mu}, X_{v}\right)} \leq C_{\mu, v, T} t^{\mu-v},
\end{array}\right.
$$

for every $t \in(0, T]$.

Example 2.1. If $A$ is a sectorial operator on $X$, then Hypotheses 2.1 are satisfied if we set $X_{\alpha}:=D_{A}(\alpha, \infty)$ for every $\alpha \in(0,2)$. Hypotheses 2.1 are satisfied also when $A$ is a negative sectorial operator and $X_{\alpha}:=D\left((-A)^{\alpha}\right)$ for every $\alpha \in(0,2)$. More generally, if $A$ is a sectorial operator, then Hypotheses 2.1 are satisfied with $X_{\alpha}:=[X, D(A)]_{\alpha}$ for every $\alpha \in(0,1), X_{1}=D(A)$ and $X_{\alpha}=\left\{x \in D(A): A x \in X_{\alpha-1}\right\}$ if $\alpha \in(1,2)$. We refer the reader also to Sect. 3.1 for another choice of the spaces $X_{\alpha}$, which guarantees the validity of a part of Hypotheses 2.1 .

We now introduce some operators which will be used extensively in this paper. 
Definition 2.1. Let $a$ and $b$ be two real numbers with $a<b$. Then, the operators $\delta, \delta_{S}: C([a, b] ; X) \rightarrow C\left([a, b]_{<}^{2} ; X\right)$ are defined as follows:

$$
\begin{aligned}
& (\delta f)(s, t)=f(t)-f(s), \\
& \left(\delta_{S} f\right)(s, t)=f(t)-f(s)-\mathfrak{a}(s, t) f(s),
\end{aligned}
$$

for every $(s, t) \in[a, b]_{<}^{2}$ and $f \in C([a, b] ; X)$, where $\mathfrak{a}(s, t)=S(t-s)-I$.

Remark 2.1. We stress that the continuity of the function $\mathfrak{a}$ in $[a, b]_{<}^{2}$ is implied by the strong continuity of the semigroup $(S(t))_{t \geq 0}$ in $(0,+\infty)$. No continuity assumption at $t=0$ is required.

\subsection{Function spaces}

Definition 2.2. For every $a, b \in \mathbb{R}$, with $a<b$ and $\alpha, \beta \in[0,2)$, we denote by:

(i) $C_{\beta}\left([a, b]_{<}^{2} ; X_{\alpha}\right)$ the subspace of $C\left([a, b]_{<}^{2} ; X_{\alpha}\right)$ consisting of functions $f$ such that

$$
\|f\|_{C_{\beta}\left([a, b]_{<}^{2} ; X_{\alpha}\right)}:=\sup _{(s, t) \in[a, b]_{<}^{2}} \frac{\|f(s, t)\|_{X_{\alpha}}}{|t-s|^{\beta}}<+\infty ;
$$

(ii) $E_{\beta}\left([a, b] ; X_{\alpha}\right)$ the subset of $C\left([a, b] ; X_{\alpha}\right)$ consisting of functions $f$ such that $\delta_{S} f \in C_{\beta}\left([a, b]_{<}^{2} ; X_{\alpha}\right)$ endowed with the norm

$$
\|f\|_{E_{\beta}\left([a, b] ; X_{\alpha}\right)}:=\|f\|_{C\left([a, b] ; X_{\alpha}\right)}+\left\|\delta_{S} f\right\|_{C_{\beta}\left([a, b]_{<}^{2} ; X_{\alpha}\right)} .
$$

Remark 2.2. For every $a, b \geq 0$ with $a<b$, and $\alpha, \beta, k \in[0,2)$ the following properties hold true.

(i) If $f \in C\left([a, b] ; X_{\alpha}\right) \cap E_{k}\left([a, b] ; X_{\beta}\right)$ then $f \in C^{\rho}\left([a, b] ; X_{\gamma}\right)$ for every $\gamma \in$ $[0, \beta]$, such that $\gamma<\alpha$, and $\rho:=\min \{k, \alpha-\gamma\}$. Indeed, for every $(s, t) \in$ $[a, b]_{<}^{2}$ we can estimate

$$
\|f(t)-f(s)\|_{X_{\gamma}} \leq\left\|\left(\delta_{S} f\right)(s, t)\right\|_{X_{\gamma}}+\|\mathfrak{a}(s, t) f(s)\|_{X_{\gamma}} .
$$

Estimating separately the two terms we get

$$
\begin{aligned}
\left\|\left(\delta_{S} f\right)(s, t)\right\|_{X_{\gamma}} & \leq K_{\beta, \gamma}\|f\|_{E_{k}\left([a, b] ; X_{\beta}\right)}|t-s|^{k} \\
\|\mathfrak{a}(s, t) f(s)\|_{X_{\gamma}} & \leq C_{\alpha, \gamma, b}\|f\|_{C\left([a, b] ; X_{\alpha}\right)}|t-s|^{\alpha-\gamma}
\end{aligned}
$$

for every $a \leq s<t \leq b$, which yields the assertion. In particular, $E_{\alpha}\left([a, b] ; X_{\alpha}\right)$ is continuously embedded into $C^{\alpha-\gamma}\left([a, b] ; X_{\gamma}\right)$ if $\alpha \in(0,1)$ and $\gamma \in[0, \alpha]$, it is contained in the space of Lipschitz continuous functions over $[a, b]$ with values in $X$ if $\alpha=1$, and it consists of constant functions if $\alpha>1$.

(ii) For every $f:[a, b]_{<}^{2} \rightarrow X$ and $\alpha, \beta, \gamma \geq 0$, such that $\beta>\gamma$, it holds that

$$
\|f\|_{C_{\gamma}\left([a, b]_{<}^{2} ; X_{\alpha}\right)} \leq|b-a|^{\beta-\gamma}\|f\|_{C_{\beta}\left([a, b]_{<}^{2} ; X_{\alpha}\right)} .
$$


Assume that $\|f\|_{C_{\beta}\left([a, b]_{<}^{2} ; X_{\alpha}\right)}$ is finite. Then, for any $s, t \in[a, b]$ with $s<t$ we can estimate

$$
\frac{\|f(s, t)\|_{X_{\alpha}}}{|t-s|^{\gamma}}=\frac{\|f(s, t)\|_{X_{\alpha}}}{|t-s|^{\beta}}|t-s|^{\beta-\gamma} \leq|b-a|^{\beta-\gamma} \frac{\|f(s, t)\|_{X_{\alpha}}}{|t-s|^{\beta}} .
$$

By taking the supremum over $(s, t) \in[a, b]_{<}^{2},(2.2)$ follows.

We recall some relevant results from [6] and [9]. In particular, we recall the definition of the Young integrals

$$
\int_{s}^{t} f(r) \mathrm{d} x(r), \quad \int_{s}^{t} S(t-r) f(r) \mathrm{d} x(r), \quad s, t \in[a, b],
$$

where $f:[a, b] \rightarrow X$ and $x:[a, b] \rightarrow \mathbb{R}$ satisfy suitable assumptions. In particular, we assume the following condition on $x$.

Hypothesis 2.2. $x \in C^{\eta}([a, b])$ for some $\eta \in(1 / 2,1)$.

Theorem 2.1. (Section 3 in [6] and Section 2 in [9]) Fix $f \in C^{\alpha}([a, b] ; X)$, where $\alpha \in(1-\eta, 1)$. Then, for each $(s, t) \in[a, b]_{<}^{2}$ the Riemann sum

$$
\sum_{i=0}^{n-1} f\left(t_{i}\right)\left(x\left(t_{i+1}\right)-x\left(t_{i}\right)\right)
$$

where $\Pi(s, t):=\left\{t_{0}=s<t_{1}<\ldots<t_{n}=t\right\}$ is a partition of $[s, t]$ and $|\Pi(s, t)|:=$ $\max \left\{t_{i+1}-t_{i}: i:=0, \ldots, n-1\right\}$, converges in $X$ as $|\Pi(s, t)|$ tends to 0 . Further, there exists a function $\mathscr{R}_{f}:[a, b]_{<}^{2} \rightarrow X$ such that

$$
\mathscr{I}_{f}(s, t):=\lim _{|\Pi(s, t)| \rightarrow 0} \sum_{i=0}^{n-1} f\left(t_{i}\right)\left(x\left(t_{i+1}\right)-x\left(t_{i}\right)\right)=f(s)(x(t)-x(s))+\mathscr{R}_{f}(s, t)
$$

for each $(s, t) \in[a, b]_{<}^{2}$, and

$$
\left\|\mathscr{R}_{f}\right\|_{C_{\eta+\alpha}\left([a, b]_{<}^{2} ; X\right)} \leq \frac{1}{1-2^{-(\eta+\alpha-1)}}\|\delta f\|_{C_{\alpha}\left([a, b]_{<}^{2} ; X\right)}\|x\|_{C^{\eta}([a, b])} .
$$

In particular,

$\left\|\mathscr{I}_{f}\right\|_{C_{\eta}\left([a, b]_{<}^{2} ; X\right)} \leq\left(\|f\|_{C([a, b] ; X)}+\frac{(b-a)^{\alpha}}{1-2^{-(\eta+\alpha-1)}}\|\delta f\|_{C_{\alpha}\left([a, b]_{<}^{2} ; X\right)}\right)\|x\|_{C^{\eta}([a, b])}$.

Remark 2.3. For each $s, \tau, t \in[a, b]$, with $s<\tau<t$, it holds that

$$
\mathscr{I}_{f}(s, t)=\mathscr{I}_{f}(s, \tau)+\mathscr{I}_{f}(\tau, t) .
$$


To check this formula it suffices to choose a family of partitions $\Pi(s, t)$ such that $\tau \in \Pi(s, t)$ and letting $|\Pi(s, t)|$ tend to 0 . As a byproduct, if we set $\Phi(t):=\mathscr{I}_{f}(a, t)$, $t \in(a, b]$, we deduce that $(\delta \Phi)(s, t)=\mathscr{I}_{f}(s, t)$. Indeed, from (2.6) we infer

$$
(\delta \Phi)(s, t)=\mathscr{I}_{f}(a, t)-\mathscr{I}_{f}(a, s)=\mathscr{I}_{f}(s, t) .
$$

Moreover, $\Phi$ is the unique function such that $\Phi(a)=0$ and

$$
\|(\delta \Phi)(t, s)-f(s)(\delta x)(t, s)\|_{X} \leq c|t-s|^{\alpha+\eta}
$$

for every $(s, t) \in[a, b]_{<}^{2}$ and some positive constant $c$.

Remark 2.4. Clearly, when $x \in C^{1}([a, b])$ the limit in (2.3) coincides with the Riemann-Stieltjes integral over the interval $[s, t]$ of the function $f$ with respect to the function $x$.

Remark 2.4 yields the following definition (see [9]).

Definition 2.3. For every $f \in C^{\alpha}([0, T])(\alpha \in(1-\eta, 1))$ and every $(s, t) \in[a, b]_{<}^{2}$, $\mathscr{I}_{f}(s, t)$ is the Young integral of $f$ in $[s, t]$ and is denoted by

$$
\int_{s}^{t} f(u) \mathrm{d} x(u)
$$

The above result reports for the construction of the "classical" Young integral. The following one, proved in [9, Sections $3 \& 4]$, accounts the construction of Young type convolutions with the semigroup $(S(t))_{t \geq 0}$.

Theorem 2.2. For each $f \in E_{k}\left([a, b] ; X_{\beta}\right)$, such that $\beta \in[0,2)$ and $\eta+k>1$, the limit

$$
\lim _{|\Pi(s, t)| \rightarrow 0} \sum_{i=0}^{n-1} S\left(t-t_{i}\right) f\left(t_{i}\right)\left(x\left(t_{i+1}\right)-x\left(t_{i}\right)\right)
$$

exists in $X$ for every $(s, t) \in[a, b]_{<}^{2}$. Further, there exists a function $\mathscr{R}_{S f}:[a, b]_{<}^{2} \rightarrow$ $X$ such that

$$
\begin{aligned}
\mathscr{I}_{S f}(s, t):= & \lim _{|\Pi(s, t)| \rightarrow 0} \sum_{i=0}^{n-1} S\left(t-t_{i}\right) f\left(t_{i}\right)\left(x\left(t_{i+1}\right)-x\left(t_{i}\right)\right) \\
& =S(t-s) f(s)(x(t)-x(s))+\mathscr{R}_{S f}(s, t),
\end{aligned}
$$

for each $(s, t) \in[a, b]_{<}^{2}$, and for each $\varepsilon \in[0,1)$ there exists a positive constant $c=c(\eta+\alpha, \varepsilon)$ such that

$$
\left\|\mathscr{R}_{S f}\right\|_{C_{\eta+k-\varepsilon}\left([a, b]_{<}^{2} ; X_{\beta+\varepsilon}\right)} \leq c\left\|\delta_{S} f\right\|_{C_{k}\left([a, b]_{<}^{2} ; X_{\beta}\right)}\|x\|_{C^{\eta}([a, b])} .
$$

In particular,

$$
\begin{aligned}
\left\|\mathscr{I}_{S f}\right\|_{C_{\eta}\left([a, b]_{<}^{2} ; X_{\beta}\right)} & \leq M_{0, \beta, b}\|f\|_{C\left([a, b] ; X_{\beta}\right)}\|x\|_{C^{\eta}([a, b])}+\left\|\mathscr{R}_{S f}\right\|_{C_{\eta}\left([a, b]_{<}^{2} ; X_{\beta}\right)} \\
& \leq\left(M_{0, \beta, b}\|f\|_{C\left([a, b] ; X_{\beta}\right)}+c(k, a, b)\left\|\delta_{S} f\right\|_{C_{k}\left([a, b]_{<}^{2} ; X_{\beta}\right)}\right)\|x\|_{C^{\eta}([a, b])} .
\end{aligned}
$$


Remark 2.5. Actually, in [9], Theorem 2.2 has been proved assuming that $X_{\beta}=$ $D\left((-A)^{\beta}\right)$. A direct inspection of the proof of [9, Theorem 4.1(2)] shows that the assertion holds true also under our assumptions, since estimates (2.1) allow us to repeat verbatim the same arguments in the quoted paper.

Again, when $x \in C^{1}([a, b])$ the limit in (2.7) coincides with the Riemann-Stieltjes integral of the function $S(t-\cdot) f$ with respect to the function $x$ over the interval $[s, t]$. As above, this remark inspires the following definition (see again [9]).

Definition 2.4. For every $f \in E_{k}\left([a, b] ; X_{\beta}\right)$, with $k \in(1-\eta, 1)$ and $\beta \in[0,2)$, $\mathscr{I}_{S f}(s, t)$ is the Young convolution of the function $S(t-\cdot) f$ with respect to $x$ in $[s, t]$ for every $(s, t) \in[a, b]_{<}^{2}$ and it is denoted by

$$
\int_{s}^{t} S(t-u) f(u) \mathrm{d} x(u) .
$$

For further use, we prove a slight extension of the estimate in [9, Theorem 4.1(2)].

Lemma 2.1. Let $f$ be a function in $E_{k}\left([a, b] ; X_{\beta}\right) \cap C\left([a, b] ; X_{\beta_{1}}\right)$ and assume that $k \in(1-\eta, 1)$ and $\beta, \beta_{1} \in[0,2)$. Then, for every $r \in[k, 1)$ the function $\mathscr{I}_{S f}$ belongs to $C_{\eta+k-r}\left([a, b]_{<}^{2} ; X_{v_{r}}\right)$, where $v_{r}:=\min \left\{r+\beta, r+\beta_{1}-k\right\}$. Further,

$$
\begin{aligned}
& \left\|\mathscr{I}_{S f}\right\|_{C_{\eta+k-r}\left([a, b]_{<}^{2} ; X_{v_{r}}\right)} \\
& \quad \leq C_{\beta_{1}, \eta, r, k}\|x\|_{C^{\eta}([a, b])}\left(\left\|\delta_{S} f\right\|_{C_{k}\left([a, b]_{<}^{2} ; X_{\beta}\right)}+\|f\|_{C\left([a, b] ; X_{\beta_{1}}\right)}\right)
\end{aligned}
$$

for every $r \in[k, 1)$.

Proof. From Theorem 2.2 it follows that $\mathscr{I}_{S f}$ is well-defined as Young convolution and

$$
\left(\mathscr{I}_{S f}\right)(s, t)=(x(t)-x(s)) S(t-s) f(s)+\mathscr{R}_{S f}(s, t), \quad(s, t) \in[a, b]_{<}^{2} .
$$

Using condition (2.1)(ii)(a), we get

$$
\begin{aligned}
& \|(x(t)-x(s)) S(t-s) f(s)\|_{X_{\gamma+\beta_{1}}} \leq[x]_{C^{\eta}([a, b])}|t-s|^{\eta}\|S(t-s) f\|_{X_{\gamma+\beta_{1}}} \\
& \quad \leq M_{\beta_{1}, \gamma+\beta_{1}, b}[x]_{C^{\eta}([a, b])}\|f\|_{C\left([a, b] ; X_{\beta_{1}}\right)}|t-s|^{\eta-\gamma}
\end{aligned}
$$

for each $(s, t) \in[a, b]_{<}^{2}, \gamma \in[0, \eta)$.

Now, we fix $r \in[k, 1)$ and take $\gamma=r-k$. Since $\eta+k>1$ it follows that $\gamma<1-k<\eta$ and $\eta-\gamma=\eta+k-r$. From (2.8) and (2.11) we conclude that $\mathscr{I}_{S f} \in C_{\eta+k-r}\left([a, b]_{<}^{2} ; X_{v_{r}}\right)$, where $v_{r}:=\min \left\{r+\beta, r+\beta_{1}-k\right\}$, and estimate (2.10) follows.

Remark 2.6. From the definition of the Young convolution it follows that if $x, x_{1}, x_{2}$ belong to $C^{\eta}([a, b])$ and $f, f_{1}, f_{2}$ belong to $E_{k}\left([a, b] ; X_{\beta}\right)$, for some $\eta \in(1 / 2,1)$, $k \in(1-\eta, 1)$ and $\beta \in[0,2)$, then

$$
\begin{aligned}
\int_{s}^{t} S(t-u) f(u) d\left(x_{1}+x_{2}\right)(u)= & \int_{s}^{t} S(t-u) f(u) \mathrm{d} x_{1}(u) \\
& +\int_{s}^{t} S(t-u) f(u) \mathrm{d} x_{2}(u)
\end{aligned}
$$


and

$$
\begin{aligned}
\int_{s}^{t} S(t-u)\left(f_{1}(u)+f_{2}(u)\right) \mathrm{d} x(u)= & \int_{s}^{t} S(t-u) f_{1}(u) \mathrm{d} x(u) \\
& +\int_{s}^{t} S(t-u) f_{2}(u) \mathrm{d} x(u)
\end{aligned}
$$

for every $(s, t) \in[a, b]_{<}^{2}$.

Now, we prove that the Young convolution (2.9) can be split into the sum of two terms.

Lemma 2.2. For every $f \in E_{k}\left([a, b] ; X_{\beta}\right)$, with $\beta \in[0,2)$ and $k \in(1-\eta, 1)$, every $(s, t) \in[a, b]_{<}^{2}$ and $\tau \in[s, t]$, it holds that

$$
\begin{aligned}
\int_{S}^{t} S(t-r) f(r) \mathrm{d} x(r) & =S(t-\tau) \int_{S}^{\tau} S(\tau-r) f(r) \mathrm{d} x(r) \\
& +\int_{\tau}^{t} S(t-r) f(r) \mathrm{d} x(r) .
\end{aligned}
$$

Proof. The proof is straightforward: it is enough to take into account the properties of Young convolution and the semigroup property of $(S(t))_{t \geq 0}$.

Corollary 2.1. For every $f \in E_{k}\left([a, b] ; X_{\beta}\right)$, with $k+\eta>1$ and $\beta \in[0,2)$, it holds that

$$
\left(\delta_{S} \mathscr{I}_{S f}(a, \cdot)\right)(s, t)=\mathscr{I}_{S f}(s, t)=\int_{s}^{t} S(t-r) f(r) \mathrm{d} x(r), \quad(s, t) \in[a, b]_{<}^{2} .
$$

Proof. From the definition of $\delta_{S}$ and of $\mathscr{I}_{S f}$ it follows that

$$
\left(\delta_{S} \mathscr{I}_{S f}(a, \cdot)\right)(s, t)=\int_{a}^{t} S(t-r) f(r) \mathrm{d} x(r)-S(t-s) \int_{a}^{s} S(s-r) f(r) \mathrm{d} x(r)
$$

for every $(s, t) \in[a, b]_{<}^{2}$. Applying Lemma 2.2 with $s=a$ and $\tau=s$ we infer that

$$
\int_{a}^{t} S(t-r) f(r) \mathrm{d} x(r)=S(t-s) \int_{a}^{s} S(s-r) f(r) \mathrm{d} x(r)+\int_{S}^{t} S(t-r) f(r) \mathrm{d} x(r),
$$

which combined with (2.14) yields the assertion.

\section{Smoothness of mild solutions}

We consider the following assumptions on the nonlinear term $\sigma$. 
Hypothesis 3.1. The function $\sigma: X \rightarrow X$ is Fréchet differentiable with bounded and locally Lipschitz continuous Fréchet derivative. Moreover, the restriction of $\sigma$ to $X_{\alpha}$ maps this space into itself for some $\alpha \in(0,1)$ such that $\alpha+\eta>1$, it is locally Lipschitz continuous and there exists a positive constant $L_{\sigma}^{\alpha}$ such that

$$
\|\sigma(x)\|_{X_{\alpha}} \leq L_{\sigma}^{\alpha}\left(1+\|x\|_{X_{\alpha}}\right), \quad x \in X_{\alpha} .
$$

Hereafter, we assume that Hypothesis 2.2 with $a=0$ and $b=T>0$ and Hypothesis 3.1 hold true.

We consider the following nonlinear Young equation

$$
\left\{\begin{array}{l}
\mathrm{d} y(t)=A y(t) \mathrm{d} t+\sigma(y(t)) \mathrm{d} x(t), \quad t \in(0, T] \\
y(0)=\psi
\end{array}\right.
$$

and we are interested in its mild solutions which take values in $D(A)$, where by mild solution we mean a function $y:[0, T] \rightarrow X$ such that $\sigma \circ y \in E_{\alpha}([0, T] ; X)$, $\eta+\alpha>1$ and

$$
y(t)=S(t) \psi+\left(\mathscr{I}_{S(\sigma \circ y)}\right)(0, t), \quad t \in[0, T] .
$$

Theorem 3.1. Let Hypotheses 2.1, 2.2 and 3.1 be satisfied, with $[a, b]=[0, T]$. Then, for every $\psi \in X_{\alpha}$ such that $\alpha \in(0,1 / 2)$ and $\eta+\alpha>1$, there exists a unique mild solution $y \in E_{\alpha}\left([0, T] ; X_{\alpha}\right)$ to equation (3.2). The solution $y$ is actually smoother since for every $a \in(0, T)$ and $\gamma \in[\eta+\alpha-1, \eta+\alpha)$, y belongs to $E_{\eta+\alpha-\gamma}\left([a, T] ; X_{\gamma}\right)$. Moreover, for every $\mu \in[0, \eta+\alpha-1)$ and $\varepsilon>0$ there exists a positive constant $c=c(\varepsilon, \mu)$ such that

$$
\|y(t)\|_{X_{1+\mu}} \leq c t^{\eta+\alpha-2-\mu-\varepsilon}, \quad t \in(0, T] .
$$

In particular, $y(t)$ belongs to $D(A)$ for every $t \in(0, T]$ and $y \in C_{\eta-\beta}\left([a, T]_{<}^{2} ; X_{\alpha+\beta}\right)$ for every $a \in(0, T)$ and $\beta \in[0, \eta)$.

The proof follows the lines of [9, Theorem 4.3], but our assumptions are weaker. In particular, in [9] the authors assume that $\eta>2 \alpha$, while we do not need this condition.

Before proving Theorem 3.1, we state the following lemma, which is a straightforward consequence of Lemma 2.2.

Lemma 3.1. Suppose that $y$ is a mild solution to (3.2). Then, for every $\tau \in[0, T]$ it holds that

$$
y(t)=S(t-\tau) y(\tau)+\int_{\tau}^{t} S(t-r) \sigma(y(r)) \mathrm{d} x(r), \quad t \in[\tau, T] .
$$

Proof of Theorem 3.1. We split the proof into some steps. 
Step 1. Here, we prove an apriori estimate. Namely, we show that if $y \in E_{\alpha}([0, T]$; $X_{\alpha}$ ) is a mild solution to (3.2), then there exists a positive constant $\Re$, which depends only on $\psi, T, \alpha, x, \eta$ and $\sigma$, such that

$$
\|y\|_{E_{\alpha}\left([0, T] ; X_{\alpha}\right)} \leq \Re .
$$

Let us fix $a, b \in[0, T]$, with $a<b$. Taking Corollary 2.1 into account, it is easy to check that $\left(\delta_{S} y\right)(s, t)=\left(\mathscr{I}_{S(\sigma \circ y)}\right)(s, t)$ for every $(s, t) \in[0, T]_{<}^{2}$. Hence, to estimate $\left\|\delta_{S} y\right\|_{C_{\alpha}\left([a, b]_{<}^{2} ; X_{\alpha}\right)}$ we can take advantage of Lemma 2.1. For this purpose, let us prove that $\sigma \circ y$ belongs to $E_{\alpha}([a, b] ; X) \cap C\left([a, b] ; X_{\alpha}\right)$. The condition $\sigma \circ y \in C\left([a, b] ; X_{\alpha}\right)$ follows immediately from (3.1), which also shows that

$$
\|\sigma \circ y\|_{C\left([a, b] ; X_{\alpha}\right)} \leq L_{\sigma}^{\alpha}\left(1+\|y\|_{C\left([a, b] ; X_{\alpha}\right)}\right) .
$$

Further, we note that the function $\delta_{S}(\sigma \circ y)$ is continuous in $[0, T]$ with values in $X$. Indeed, fix $\left(t_{0}, s_{0}\right) \in[a, b]_{<}^{2}$. Then,

$$
\begin{aligned}
&\left\|\left(\delta_{S}(\sigma \circ y)\right)(t, s)-\left(\delta_{S}(\sigma \circ y)\right)\left(t_{0}, s_{0}\right)\right\|_{X} \\
& \quad \leq \|\left(\sigma(y(t))-\left(\sigma\left(y\left(t_{0}\right)\right)\left\|_{X}+\right\| S(t-s) \sigma(y(s))-S\left(t_{0}-s_{0}\right) \sigma\left(y\left(s_{0}\right)\right) \|_{X}\right.\right. \\
& \leq L\left\|y(t)-y\left(t_{0}\right)\right\|_{X}+\|S(t-s)\|_{\mathscr{L}(X)}\left\|\sigma(y(s))-\sigma\left(y\left(s_{0}\right)\right)\right\|_{X} \\
& \quad+\left\|\left(S(t-s)-S\left(t_{0}-s_{0}\right)\right) \sigma\left(y\left(s_{0}\right)\right)\right\|_{X} \\
& \leq L\left\|y(t)-y\left(t_{0}\right)\right\|_{X}+L M_{0,0, T}\left\|y(s)-y\left(s_{0}\right)\right\|_{X} \\
& \quad+2 C_{\alpha, 0, T}\left\|\sigma\left(y\left(s_{0}\right)\right)\right\|_{X_{\alpha}}\left|t-t_{0}\right|^{\alpha}
\end{aligned}
$$

for every $(t, s) \in[0, T]_{<}^{2}$, where $M_{0,0, T}$ and $C_{\alpha, 0, T}$ are the constants in condition (2.1), $L$ denotes the Lipschitz constant of $\sigma$ on $X$, and the last side of the previous chain of inequalities vanishes as $(t, s)$ tends to $\left(t_{0}, s_{0}\right)$. Next, we split

$$
\left(\delta_{S}(\sigma \circ y)\right)(s, t)=(\delta(\sigma \circ y))(s, t)-\mathfrak{a}(s, t) \sigma(y(s)), \quad(s, t) \in[0, T]_{<}^{2} .
$$

and estimate separately the two terms. As far as the first one is considered, we observe that

$$
\begin{aligned}
\|(\delta(\sigma \circ y))(s, t)\|_{X} & =\|\sigma(y(t))-\sigma(y(s))\|_{X} \\
& \leq L_{\sigma}\|y(t)-y(s)\|_{X} \\
& \leq L_{\sigma}\left(\left\|\left(\delta_{S} y\right)(s, t)\right\|_{X}+\|\mathfrak{a}(s, t) y(s)\|_{X}\right) \\
& \leq L_{\sigma}\left(1+C_{\alpha, 0, T}\right)\|y\|_{E_{\alpha}\left([a, b] ; X_{\alpha}\right)}|t-s|^{\alpha}
\end{aligned}
$$

for every $(s, t) \in[a, b]_{<}^{2}$, where $L_{\sigma}$ denotes the Lipschitz constant of the function $\sigma$. As far as the term $\mathfrak{a}(s, t) \sigma(y(s))$ is concerned, we use (3.1) to estimate

$$
\begin{aligned}
\|\mathfrak{a}(s, t) \sigma(y(s))\|_{X} & \leq C_{\alpha, 0, T}\|\sigma(y(s))\|_{X_{\alpha}}|t-s|^{\alpha} \\
& \leq C_{\alpha, 0, T} L_{\sigma}^{\alpha}\left(1+\|y\|_{E_{\alpha}\left([a, b] ; X_{\alpha}\right)}\right)|t-s|^{\alpha}
\end{aligned}
$$


for every $(s, t) \in[a, b]_{<}^{2}$. We have so proved that $\sigma \circ y \in E_{\alpha}([a, b] ; X)$ and

$$
\|\left(\delta_{S}(\sigma \circ y) \|_{C_{\alpha}\left([a, b]_{<}^{2} ; X\right)} \leq\left(L_{\sigma}+L_{\sigma}^{\alpha}\right)\left(1+C_{\alpha, 0, T}\right)\left(1+\|y\|_{E_{\alpha}\left([a, b] ; X_{\alpha}\right)}\right) .\right.
$$

Thus, we can apply Lemma 2.1 as claimed, with $k=\beta_{1}=\alpha$ and $\beta=0$, to infer that $\mathscr{I}_{S(\sigma \circ y)}$ belongs to $C_{\eta+\alpha-r}\left([a, b]_{<}^{2} ; X_{r}\right)$ for every $r \in[\alpha, 1)$ and

$$
\begin{aligned}
& \left\|\mathscr{I}_{S(\sigma \circ y)}\right\|_{C_{\eta+\alpha-r}\left([a, b]_{<}^{2} ; X_{r}\right)} \\
& \quad \leq C_{\alpha, \eta, r, \alpha}\|x\|_{C^{\eta}([0, T])}\left(\left\|\delta_{S}(\sigma \circ y)\right\|_{C_{\alpha}\left([a, b]_{<}^{2} ; X\right)}+\|\sigma \circ y\|_{C\left([a, b] ; X_{\alpha}\right)}\right) \\
& \quad \leq C_{\alpha, \eta, r, \alpha}\|x\|_{C^{\eta}([0, T])}\left(L_{\sigma}+L_{\sigma}^{\alpha}\right)\left(2+C_{\alpha, 0, T}\right)\left(1+\|y\|_{E_{\alpha}\left([a, b] ; X_{\alpha}\right)}\right) .
\end{aligned}
$$

Since $\alpha<1 / 2<\eta$, it follows that

$$
\left\|\mathscr{I}_{S(\sigma \circ y)}\right\|_{C_{\alpha}\left([a, b]_{<}^{2} ; X_{\alpha}\right)} \leq(b-a)^{\eta-\alpha}\left\|\mathscr{I}_{S(\sigma \circ y)}\right\|_{C_{\eta}\left([a, b]_{<}^{2} ; X_{\alpha}\right)},
$$

so that, applying (3.11) with $r=\alpha$, we conclude that

$$
\begin{aligned}
\left\|\delta_{S} y\right\|_{C_{\alpha}\left([a, b]_{<}^{2} ; X_{\alpha}\right)} & =\left\|\mathscr{I}_{S(\sigma \circ y)}\right\|_{C_{\alpha}\left([a, b]_{<}^{2} ; X_{\alpha}\right)} \\
& \leq \mathfrak{C}(b-a)^{\eta-\alpha}\|x\|_{C^{\eta}([0, T])}\left(1+\|y\|_{E_{\alpha}\left([a, b] ; X_{\alpha}\right)}\right),
\end{aligned}
$$

where $\mathfrak{C}:=C_{\alpha, \eta, r, \alpha}\left(L_{\sigma}+L_{\sigma}^{\alpha}\right)\left(2+C_{\alpha, 0, T}\right)$. Further, from (3.5) with $\tau=a, t \in[a, b]$ and Corollary 2.1, we get

$$
\begin{aligned}
\|y\|_{C\left([a, b] ; X_{\alpha}\right)} & \leq M_{\alpha, \alpha, b}\|y(a)\|_{X_{\alpha}}+\left\|\left(\delta_{S} y\right)(a, \cdot)\right\|_{C\left([a, b] ; X_{\alpha}\right)} \\
& \leq M_{\alpha, \alpha, T}\|y(a)\|_{X_{\alpha}}+(b-a)^{\alpha}\left\|\delta_{S} y\right\|_{C_{\alpha}\left([a, b]_{<}^{2} ; X_{\alpha}\right)} \\
& \leq M_{\alpha, \alpha, T}\|y(a)\|_{X_{\alpha}}+\mathfrak{C}(b-a)^{\eta}\|x\|_{C^{\eta}([0, T])}\left(1+\|y\|_{E_{\alpha}\left([a, b] ; X_{\alpha}\right)}\right) .
\end{aligned}
$$

Taking (3.12) and (3.13) into account, this gives

$$
\begin{aligned}
\|y\|_{E_{\alpha}\left([a, b] ; X_{\alpha}\right) \leq} & M_{\alpha, \alpha, T}\|y(a)\|_{X_{\alpha}} \\
& +\mathfrak{C}(b-a)^{\eta-\alpha}\left(1+(b-a)^{\alpha}\right)\|x\|_{C^{\eta}([0, T])}\left(1+\|y\|_{E_{\alpha}\left([a, b] ; X_{\alpha}\right)}\right) \\
\leq & M_{\alpha, \alpha, T}\|y(a)\|_{X_{\alpha}} \\
& +\mathfrak{C}(b-a)^{\eta-\alpha}\left(1+T^{\alpha}\right)\|x\|_{C^{\eta}([0, T])}\left(1+\|y\|_{E_{\alpha}\left([a, b] ; X_{\alpha}\right)}\right) .
\end{aligned}
$$

Let us set

$$
\bar{T}=\left(\frac{1}{2 \mathfrak{C}\left(1+T^{\alpha}\right)\|x\|_{C^{\eta}([0, T])}}\right)^{\frac{1}{\eta-\alpha}}
$$

If $b-a \leq \bar{T}$, then we get

$$
\|y\|_{E_{\alpha}\left([a, b] ; X_{\alpha}\right)} \leq 2 M_{\alpha, \alpha, T}\|y(a)\|_{X_{\alpha}}+1 .
$$


Now, we introduce the function $\phi:(0, \infty) \rightarrow(0, \infty)$, defined by $\phi(r)=2 M_{\alpha, \alpha, T} r+$ 1 for every $r>0$ and split

$$
[0, T]=\bigcup_{n=0}^{N-1}\left[t_{n}, t_{n+1}\right]
$$

where $0=t_{0}<t_{1}<t_{2}<\ldots<t_{N}=T$ and $t_{n+1}-t_{n} \leq \bar{T}$ for every $n=$ $0, \ldots, N-1$. From (3.15) it follows that

$$
\|y\|_{C\left(\left[t_{n}, t_{n+1}\right] ; X_{\alpha}\right)} \leq \phi\left(\left\|y\left(t_{n}\right)\right\|_{X_{\alpha}}\right) \leq \phi^{n+1}\left(\|\psi\|_{X_{\alpha}}\right)
$$

for every $n=0, \ldots, N-1$, where $\phi^{k}$ denotes the composition of $\phi$ with itself $k$ times. Since $\phi(r)>r$ for every $r>0$, from (3.16) we conclude that

$$
\|y\|_{C\left([0, T] ; X_{\alpha}\right)} \leq \phi^{N}\left(\|\psi\|_{X_{\alpha}}\right) .
$$

In particular, for each interval $[s, t] \subset[0, T]$ whose length is less than or equal to $\bar{T}$ we get

$$
\|y\|_{E_{\alpha}\left([s, t] ; X_{\alpha}\right)} \leq 2 M_{\alpha, \alpha, T} \phi^{N}\left(\|y(s)\|_{X_{\alpha}}\right)+1 \leq \phi^{N+1}\left(\|\psi\|_{X_{\alpha}}\right) .
$$

Now we are able to estimate $\left\|\delta_{S} y\right\|_{C_{\alpha}\left([0, T]_{<}^{2} ; X_{\alpha}\right)}$. We stress that, if $|t-s| \leq \bar{T}$, then from (3.15) we get

$$
\left\|\left(\delta_{S} y\right)(s, t)\right\|_{X_{\alpha}} \leq \phi^{N+1}\left(\|\psi\|_{X_{\alpha}}\right)|t-s|^{\alpha}
$$

and if $|t-s|>\bar{T}$ then

$$
\frac{\left\|\left(\delta_{S} y\right)(s, t)\right\|_{X_{\alpha}}}{|t-s|^{\alpha}} \leq \frac{\|y(t)-S(t-s) y(s)\|_{X_{\alpha}}}{\bar{T}^{\alpha}} \leq \frac{\left(1+M_{\alpha, \alpha, T}\right) \phi^{N}\left(\|\psi\|_{X_{\alpha}}\right)}{\bar{T}^{\alpha}} .
$$

From (3.17), (3.18) and (3.19) it follows that

$$
\begin{aligned}
\|y\|_{E_{\alpha}\left([0, T] ; X_{\alpha}\right) \leq} & \phi^{N}\left(\|\psi\|_{X_{\alpha}}\right) \\
& +\max \left\{\phi^{N+1}\left(\|\psi\|_{X_{\alpha}}\right), \bar{T}^{-\alpha}\left(1+M_{\alpha, \alpha, T}\right) \phi^{N}\left(\|\psi\|_{X_{\alpha}}\right)\right\} \\
& =\Re .
\end{aligned}
$$

Step 2. Here, we prove that there exists a unique mild solution to Eq. (3.2). For this purpose, we introduce the operator $\Gamma_{1}: E_{\alpha}\left(\left[0, T_{*}\right] ; X_{\alpha}\right) \rightarrow E_{\alpha}\left(\left[0, T_{*}\right] ; X_{\alpha}\right)$, defined by $\left(\Gamma_{1}(y)\right)(t)=S(t) \psi+\mathscr{I}_{S(\sigma \circ y)}(0, t)$ for every $t \in\left[0, T_{*}\right]$ and $\left(\Gamma_{1}(y)\right)(0)=\psi$, where $T_{*} \in(0, T]$ has to be properly chosen later on. We are going to prove that $\Gamma_{1}$ is a contraction in $\mathcal{B}=\left\{y \in E_{\alpha}\left(\left[0, T_{*}\right] ; X_{\alpha}\right):\|y\|_{E_{\alpha}\left(\left[0, T_{*}\right] ; X_{\alpha}\right)} \leq 2 M_{\alpha, \alpha, T} \mathfrak{R}\right\}$. To begin 
with, we fix $y \in \mathcal{B}$ and observe that $\delta_{S} \Gamma_{1}(y)=\mathscr{I}_{S(\sigma \circ y)}$. Hence, from (3.14), we can estimate

$$
\begin{aligned}
\| & \Gamma_{1}(y) \|_{E_{\alpha}\left(\left[0, T_{*}\right] ; X_{\alpha}\right)} \\
& \leq M_{\alpha, \alpha, T}\|\psi\|_{X_{\alpha}}+\mathfrak{C} T_{*}^{\eta-\alpha}\left(1+T_{*}^{\alpha}\right)\|x\|_{C^{\eta}([0, T])}\left(1+\|y\|_{E_{\alpha}\left(\left[0, T_{*}\right] ; X_{\alpha}\right)}\right) \\
& \leq M_{\alpha, \alpha, T} \mathfrak{R}+\mathfrak{C} T_{*}^{\eta-\alpha}\left(1+T^{\alpha}\right)\|x\|_{C^{\eta}([0, T])}\left(1+2 M_{\alpha, \alpha, T} \mathfrak{R}\right) .
\end{aligned}
$$

We now choose $T_{*} \leq T$ such that $\mathfrak{C} T_{*}^{\eta-\alpha}\left(1+T^{\alpha}\right)\|x\|_{C^{\eta}([0, T])}\left(1+2 M_{\alpha, \alpha, T} \Re\right) \leq$ $M_{\alpha, \alpha, T} \mathfrak{R}$. With this choice of $T_{*}$, we conclude that $\Gamma_{1}(y)$ belongs to $\mathcal{B}$.

Let us prove that $\Gamma_{1}$ is a $1 / 2$-contraction. Fix $y_{1}, y_{2} \in \mathcal{B}$. The linearity of the Young integral gives $\left(\Gamma_{1}\left(y_{1}\right)\right)(t)-\left(\Gamma_{1}\left(y_{2}\right)\right)(t)=\mathscr{I}_{S\left(\sigma \circ y_{1}-\sigma \circ y_{2}\right)}(0, t)$ for every $t \in\left[0, T_{*}\right]$, so that we can estimate

$$
\left\|\Gamma_{1}\left(y_{1}\right)-\Gamma_{1}\left(y_{2}\right)\right\|_{X_{\alpha}} \leq T_{*}^{\eta}\left\|\mathscr{I}_{S\left(\sigma \circ y_{1}-\sigma \circ y_{2}\right)}\right\|_{C_{\eta}\left(\left[0, T_{*}\right]_{<}^{2} ; X_{\alpha}\right)}
$$

and, as in Step 1 (see the first inequality in (3.11)),

$$
\begin{aligned}
& \left\|\Gamma_{1}\left(y_{1}\right)-\Gamma_{1}\left(y_{2}\right)\right\|_{C_{\eta}\left(\left[0, T_{*}\right]_{<}^{2} ; X_{\alpha}\right)} \\
& \quad \leq C_{\alpha, \eta}\|x\|_{C^{\eta}([0, T])}\left(\left\|\delta_{S}\left(\sigma \circ y_{1}-\sigma \circ y_{2}\right)\right\|_{C_{\alpha}\left(\left[0, T_{*}\right]_{<}^{2} ; X\right)}\right. \\
& \left.\quad+\left\|\sigma \circ y_{1}-\sigma \circ y_{2}\right\|_{C\left(\left[0, T_{*}\right] ; X_{\alpha}\right)}\right) .
\end{aligned}
$$

We set $R:=2 M_{\alpha, \alpha, T} \Re \geq \max \left\{\left\|y_{1}\right\|_{C\left(\left[0, T_{*}\right] ; X_{\alpha}\right)},\left\|y_{2}\right\|_{C\left(\left[0, T_{*}\right] ; X_{\alpha}\right)}\right\}$ and note that

$$
\begin{aligned}
\left\|\mathfrak{a}(s, t)\left(\sigma\left(y_{1}(s)\right)-\sigma\left(y_{2}(s)\right)\right)\right\|_{X} & \leq C_{\alpha, 0, T}|t-s|^{\alpha}\left\|\sigma\left(y_{1}(s)\right)-\sigma\left(y_{2}(s)\right)\right\|_{X_{\alpha}} \\
& \leq C_{\alpha, 0, T} L_{\sigma}^{\alpha, R}|t-s|^{\alpha}\left\|y_{1}-y_{2}\right\|_{C\left(\left[0, T_{*}\right] ; X_{\alpha}\right)},
\end{aligned}
$$

where $L_{\sigma}^{\alpha, r}$ denotes the Lipschitz constant of the restriction of $\sigma$ to the ball $B(0, r) \subset$ $X_{\alpha}$ and we have used the condition (2.1)(b). Further, by taking advantage of the smoothness of $\sigma$ we get

$$
\begin{aligned}
& \left(\delta\left(\sigma \circ y_{1}-\sigma \circ y_{2}\right)(s, t)\right) \\
& \quad=\sigma\left(y_{1}(s)+\left(\delta y_{1}\right)(s, t)\right)-\sigma\left(y_{1}(s)\right)-\sigma\left(y_{2}(s)+\left(\delta y_{1}\right)(s, t)\right)+\sigma\left(y_{2}(s)\right) \\
& \quad+\sigma\left(y_{2}(s)+\left(\delta y_{1}\right)(s, t)\right)-\sigma\left(y_{2}(s)+\left(\delta y_{2}\right)(s, t)\right) \\
& \quad=\int_{0}^{1}\left\langle\sigma^{\prime}\left(y_{1}(s)+r\left(\delta y_{1}\right)(s, t)\right)-\sigma^{\prime}\left(y_{2}(s)+r\left(\delta y_{1}\right)(s, t)\right), \delta y_{1}(s, t)\right\rangle d r \\
& \quad+\sigma\left(y_{2}(s)+\left(\delta y_{1}\right)(s, t)\right)-\sigma\left(y_{2}(s)+\left(\delta y_{2}\right)(s, t)\right) .
\end{aligned}
$$

Since for every $s, t \in[0, \bar{T}]$, with $s<t$, and $r \in(0,1)$, it holds that

$$
\left\|y_{1}(s)+r \delta y_{1}(s, t)\right\|_{X_{\alpha}} \vee\left\|y_{2}(s)+r \delta y_{1}(s, t)\right\|_{X_{\alpha}} \leq 3 R,
$$


and recalling that $R \geq 1$, it follows that

$$
\begin{aligned}
&\left\|\delta\left(\sigma \circ y_{1}-\sigma \circ y_{2}\right)(s, t)\right\|_{X} \\
& \leq K_{\sigma^{\prime}}^{R}\left\|y_{1}-y_{2}\right\|_{C\left(\left[0, T_{*}\right] ; X\right)}\left\|\left(\delta y_{1}\right)(s, t)\right\|_{X}+L_{\sigma}\left\|\left(\delta\left(y_{1}-y_{2}\right)\right)(s, t)\right\|_{X} \\
& \leq K_{\sigma^{\prime}}^{R}\left\|y_{1}-y_{2}\right\|_{C\left(\left[0, T_{*}\right] ; X\right)}\left(\left\|\left(\delta_{S} y_{1}\right)(s, t)\right\|_{X}+\left\|\mathfrak{a}(s, t) y_{1}(s)\right\|_{X}\right) \\
& \quad+L_{\sigma}\left(\left\|\left(\delta_{S}\left(y_{1}-y_{2}\right)\right)(s, t)\right\|_{X}+\left\|\mathfrak{a}(s, t)\left(y_{1}(s)-y_{2}(s)\right)\right\|_{X}\right) \\
& \leq K_{\sigma^{\prime}}^{R}\left(\left\|y_{1}\right\|_{E_{\alpha}\left(\left[0, T_{*}\right] ; X_{\alpha}\right)}+C_{\alpha, 0, T}\left\|y_{1}\right\|_{C\left(\left[0, T_{*}\right] ; X_{\alpha}\right)}\right)\left\|y_{1}-y_{2}\right\|_{C\left(\left[0, T_{*}\right] ; X\right)}|t-s|^{\alpha} \\
& \quad+L_{\sigma}\left(\left\|y_{1}-y_{2}\right\|_{E_{\alpha}\left(\left[0, T_{*}\right] ; X_{\alpha}\right)}+C_{\alpha, 0, T}\left\|y_{1}-y_{2}\right\|_{\left.C\left[0, T_{*}\right] ; X_{\alpha}\right)}\right)|t-s|^{\alpha} \\
& \leq\left(1+C_{\alpha, 0, T}\right) R\left(K_{\sigma^{\prime}}^{R}+L_{\sigma}\right)\left\|y_{1}-y_{2}\right\|_{E_{\alpha}\left(\left[0, T_{*}\right] ; X_{\alpha}\right)|t-s|^{\alpha},}
\end{aligned}
$$

where $K_{\sigma^{\prime}}^{R}$ denotes the Lipschitz constant of the restriction of function $\sigma^{\prime}$ to the ball $B\left(3 K_{\alpha, 0} R\right) \subset X$. As far as $\left\|\sigma \circ y_{1}-\sigma \circ y_{2}\right\|_{C\left(\left[0, T_{*}\right] ; X_{\alpha}\right)}$ in (3.21) is concerned, it holds that

$$
\left\|\sigma\left(y_{1}(t)\right)-\sigma\left(y_{2}(t)\right)\right\|_{X_{\alpha}} \leq L_{\sigma}^{\alpha, R}\left\|y_{1}-y_{2}\right\|_{E_{\alpha}\left(\left[0, T_{*}\right] ; X_{\alpha}\right)}
$$

for every $t \in\left[0, T_{*}\right]$. From (3.21), (3.22), (3.24) and (3.25) we get

$$
\begin{aligned}
\left\|\Gamma_{1}\left(y_{1}\right)-\Gamma_{1}\left(y_{2}\right)\right\|_{E_{\alpha}\left(\left[0, T_{*}\right] ; X_{\alpha}\right)} & \leq T_{*}^{\eta-\alpha}\left\|\mathscr{I}_{S\left(\sigma \circ y_{1}-\sigma \circ y_{2}\right)}\right\|_{C_{\eta}\left(\left[0, T_{*}\right]_{<}^{2} ; X_{\alpha}\right)} \\
& \leq c T_{*}^{\eta-\alpha}\left\|y_{1}-y_{2}\right\|_{E_{\alpha}\left(\left[0, T_{*}\right] ; X_{\alpha}\right)}
\end{aligned}
$$

where $c$ is a positive constant which depends on $x, \alpha, R, \sigma, \eta$ but not on $T_{*}$ nor on $\psi$.

Based on (3.20) and (3.26), we can now fix $T_{*}>0$ such that $\Gamma_{1}$ is a $1 / 2$-contraction in $\mathcal{B}$. If $T_{*}=T$, then we are done. Otherwise, we use a standard procedure to extend the solution of the Young equation (3.2): we introduce the operator $\Gamma_{2}$ defined by

$$
\left(\Gamma_{2}(y)\right)(t)=S\left(t-T_{*}\right) y_{1}\left(T_{*}\right)+\mathscr{I}_{S(\sigma \circ y)}\left(T_{*}, t\right), \quad T_{*} \leq t \leq T_{* *}=\min \left\{2 T_{*}, T\right\},
$$

for every $y \in \mathcal{B}_{2}:=\left\{z \in E_{\alpha}\left(\left[T_{*}, T_{* *}\right] ; X_{\alpha}\right):\|y\|_{E_{\alpha}\left(\left[T_{*}, T_{* *}\right] ; X_{\alpha}\right)} \leq 2 M_{\alpha, \alpha, T} \mathfrak{R}\right\}$. Since $y_{1}$ is a mild solution to (3.2), from (3.6), which clearly holds true also with $T_{*}<T$ and the same constant $\mathfrak{R}$, it follows that $\left\|y_{1}\left(T_{*}\right)\right\|_{X_{\alpha}} \leq \mathfrak{R}$. Then, by the same computations as above we show that $\Gamma_{2}$ is a $1 / 2$-contraction in $\mathcal{B}_{2}$. Denote by $y_{2}$ its unique fixed point. Thanks to Lemma 2.2, the function $y$ defined by $y(t)=y_{1}(t)$ if $t \in\left[0, T_{*}\right]$ and $y(t)=y_{2}(t)$ if $t \in\left[T_{*}, T_{* *}\right]$ is a mild solution to Eq. (3.2) in [0, $\left.T_{* *}\right]$. Obviously, if $T_{* *}<T$, then we can repeat the same procedure and in a finite number of steps we extend $y$ to whole $[0, T]$. Estimate (3.6) yields also the uniqueness of the mild solution to Eq. (3.2).

Step 3. From the arguments in the first part of Step 1 (see (3.11)), we deduce that $\mathscr{I}_{S(\sigma \circ y)}$ belongs to $C_{\eta+\alpha-r}\left([0, T]_{<}^{2} ; X_{r}\right)$ for every $r \in[\alpha, 1)$. The smoothing properties of the semigroup $(S(t))_{t \geq 0}$ (see condition (2.1)(ii)(a)), estimates (3.6) and (3.11) show that $y(t) \in X_{r}$ and

$$
\begin{aligned}
\|y(t)\|_{X_{r}} & \leq\|S(t) \psi\|_{X_{r}}+\left\|\mathscr{I}_{S(\sigma \circ y)}(0, t)\right\|_{X_{r}} \\
& \leq M_{\alpha, r, T} t^{\alpha-r}\|\psi\|_{X_{\alpha}}+\left\|\mathscr{I}_{S(\sigma \circ y)}\right\|_{C_{\eta+\alpha-r}\left([0, T]_{<}^{2} ; X_{r}\right)} t^{\eta+\alpha-r} \\
& \leq c_{1}\left(1+T^{\eta}\right) t^{\alpha-r}
\end{aligned}
$$


for every $t \in(0, T]$ and some positive constant $c_{1}$, which depends on $\alpha, \eta, r$, $\|x\|_{C^{\eta}([0, T])},\|\psi\|_{X_{\alpha}}, \sigma, \mathfrak{R}$, and is a continuous function of $\|x\|_{C^{\eta}([0, T])}$ and $\mathfrak{R}$. Now, we observe that $\|y(t)-y(s)\|_{X_{r}} \leq\left\|\delta_{S} y(s, t)\right\|_{X_{r}}+\|\mathfrak{a}(s, t) y(s)\|_{X_{r}}$. Since $\delta_{S} y=\mathscr{I}_{S(\sigma \circ y)}$, from (3.11) it follows that

$$
\left\|\delta_{S} y(s, t)\right\|_{X_{r}} \leq c_{2}(t-s)^{\eta+\alpha-r}, \quad(s, t) \in[0, T]_{<}^{2},
$$

where $c_{2}=c_{2}\left(\alpha, \eta, r,\|x\|_{C^{\eta}([0, T])},\|\psi\|_{X_{\alpha}}, \sigma, \mathfrak{R}\right)$ is a positive constant, which depends in a continuous way on $\|x\|_{C^{\eta}([0, T])}$ and $\mathfrak{R}$. Moreover, using condition (2.1)(b) and estimate (3.27) (with $r$ being replaced by $r+\beta$ ), we get

$$
\begin{aligned}
\|\mathfrak{a}(s, t) y(s)\|_{X_{r}} & \leq C_{r+\beta, r, T}|t-s|^{\beta}\|y(s)\|_{X_{r+\beta}} \\
& \leq C_{r+\beta, r, T} \tilde{c}_{1}\left(1+T^{\eta}\right) s^{\alpha-r-\beta}|t-s|^{\beta},
\end{aligned}
$$

where $\beta>0$ is such that $r+\beta<1$ (such $\beta$ exists since we are assuming $r \in$ $[\alpha, 1))$. From these two last estimates it follows immediately that $y \in C\left((0, T] ; X_{r}\right)$. Moreover, for every $\varepsilon \in(0, T]$ and $r \in[\alpha, 1)$, there exists a positive constant $c_{3}=c_{3}\left(\alpha, \eta, r,\|x\|_{C^{\eta}([0, T])},\|\psi\|_{X_{\alpha}}, \sigma, \Re, T\right)$, which depends in a continuous way on $\|x\|_{C^{\eta}([0, T])}$ and on $\mathfrak{R}$, such that

$$
\|y\|_{C\left([\varepsilon, T] ; X_{r}\right)}+\left\|\delta_{S} y\right\|_{C_{\eta+\alpha-r}\left([\varepsilon, T]_{<}^{2} ; X_{r}\right)} \leq c_{3} \varepsilon^{\alpha-r} .
$$

Next, we estimate $\left\|\left(\delta_{S}(\sigma \circ y)\right)(s, t)\right\|_{X_{\lambda}}$ when $\eta+\alpha-\lambda>1$, i.e., $\lambda \in[0, \eta+\alpha-1)$. As usually, we separately estimate $\|(\delta(\sigma \circ y))(s, t)\|_{X_{\lambda}}$ and $\|\mathfrak{a}(s, t) \sigma(y(s))\|_{X_{\lambda}}$. Note that $\lambda<\alpha$ since $\eta<1$. We fix $\varepsilon>0$ and observe that the continuous embedding $X_{\alpha} \hookrightarrow X_{\lambda}$, (3.11) and (3.27) (with $r=2 \alpha-\lambda$, which belongs to [ $\alpha, 1$ ) since $\alpha<1 / 2$ ) give

$$
\begin{aligned}
\|(\delta(\sigma \circ y))(s, t)\|_{X_{\lambda} \leq} \leq & K_{\alpha, \lambda} L_{\sigma}^{\alpha, \Re}\left(\left\|\left(\delta_{S} y\right)(s, t)\right\|_{X_{\alpha}}+\|\mathfrak{a}(s, t) y(s)\|_{X_{\alpha}}\right) \\
\leq & K_{\alpha, \lambda} L_{\sigma}^{\alpha, \Re}\left(\|y\|_{E_{\alpha}\left([0, T] ; X_{\alpha}\right)}|t-s|\right. \\
& \left.+C_{2 \alpha-\lambda, \alpha, T} \varepsilon^{\lambda-\alpha}|t-s|^{\alpha-\lambda}\right) \\
\leq & c_{4} \varepsilon^{\lambda-\alpha}|t-s|^{\alpha-\lambda}
\end{aligned}
$$

for every $(s, t) \in[\varepsilon, T]_{<}^{2}$, where $c_{4}=c_{4}\left(\alpha, \eta,\|x\|_{C^{\eta}([0, T])}, \mathfrak{R}, T, \lambda\right)$. Moreover,

$$
\begin{aligned}
\|\mathfrak{a}(s, t) \sigma(y(s))\|_{X_{\lambda}} & \leq C_{\alpha, \lambda, T}\|\sigma(y(s))\|_{X_{\alpha}}|t-s|^{\alpha-\lambda} \\
& \leq C_{\alpha, \lambda, T} L_{\sigma}^{\alpha}\left(1+\|y(s)\|_{X_{\alpha}}\right)|t-s|^{\alpha-\lambda} \\
& \leq C_{\alpha, \lambda, T} L_{\sigma}^{\alpha}(1+\Re)|t-s|^{\alpha-\lambda}
\end{aligned}
$$

for every $(s, t) \in[\varepsilon, T]_{<}^{2}$. From (3.30) and (3.31), it follows that

$$
\sup _{\varepsilon \leq s<t \leq T} \frac{\left\|\left(\delta_{S}(\sigma \circ y)\right)(s, t)\right\|_{X_{\lambda}}}{|t-s|^{\alpha-\lambda}} \leq c_{4} \varepsilon^{\lambda-\alpha}+C_{\alpha, \lambda, T} L_{\sigma}^{\alpha}(1+\Re) .
$$


Moreover, arguing as in the proof of (3.8) we can show that

$$
\begin{aligned}
& \left\|\left(\delta_{S}(\sigma \circ y)\right)(t, s)-\left(\delta_{S}(\sigma \circ y)\right)\left(t_{0}, s_{0}\right)\right\|_{X_{\lambda}} \\
& \quad \leq L^{\alpha} K_{\alpha, \lambda}\left(\left\|y(t)-y\left(t_{0}\right)\right\|_{X_{\alpha}}+M_{\alpha, \alpha}\left\|y(s)-y\left(s_{0}\right)\right\|_{X_{\alpha}}\right) \\
& \quad+2 C_{\alpha, \lambda}\left\|\sigma\left(y\left(s_{0}\right)\right)\right\|_{X_{\alpha}}\left|t-t_{0}\right|^{\alpha-\lambda}
\end{aligned}
$$

for every $\left(t_{0}, s_{0}\right),(t, s) \in[\varepsilon, T]_{<}^{2}$, where $L^{\alpha}$ denotes the Lipschitz constant of $\sigma$ on the subset $\left\{y \in X_{\alpha}:\|y\|_{X_{\alpha}} \leq \sup _{t \in[0, T]}\|y(t)\|_{X_{\alpha}}\right\}$ of $X_{\alpha}$, and conclude that $\delta_{S}(\sigma \circ$ $y) \in C_{\alpha-\lambda}\left([\varepsilon, T]_{<}^{2} ; X_{\lambda}\right)$. Further, $\sigma \circ y$ belongs to $C\left([\varepsilon, T] ; X_{\alpha}\right)$. From Lemma 2.1 with $k=\alpha-\lambda, \beta=\lambda, \beta_{1}=\alpha$ and $r=\gamma$, we infer that $\mathscr{I}_{S(\sigma \circ y)}$ belongs to $C_{\eta+\alpha-\lambda-\gamma}\left([\varepsilon, T]_{<}^{2} ; X_{\gamma+\lambda}\right)$ for every $\gamma \in[\alpha-\lambda, 1)$ and

$$
\begin{aligned}
& \left\|\mathscr{I}_{S(\sigma \circ y)}\right\|_{C_{\eta+\alpha-\lambda-\gamma}\left([\varepsilon, T]_{<}^{2} ; X_{\gamma+\lambda}\right)} \\
& \quad \leq C_{\alpha, \eta, \gamma, \alpha-\lambda}\|x\|_{C^{\eta}([0, T])}\left(\|\sigma \circ y\|_{C\left([\varepsilon, T] ; X_{\alpha}\right)}+\left\|\delta_{S}(\sigma \circ y)\right\|_{C_{\alpha-\lambda}\left([\varepsilon, T]_{<}^{2} ; X_{\lambda}\right)}\right) \\
& \quad \leq c_{5} \varepsilon^{\lambda-\alpha}
\end{aligned}
$$

for some positive constant $c_{5}=c_{5}\left(\alpha, \eta, \sigma,\|x\|_{C^{\eta}([0, T])}, \mathfrak{R}, T, \lambda, \gamma,\|\psi\|_{X_{\alpha}}\right)$, which does not depend on $\varepsilon$. From (3.5), with $\tau=\varepsilon$, we can write

$$
y(t)=S(t-\varepsilon)(y(\varepsilon))+\mathscr{I}_{S(\sigma \circ y)}(\varepsilon, t), \quad t \in[\varepsilon, T]
$$

and applying (3.27), with $t=\varepsilon$ and $r=\alpha$, (3.32) and (3.33) we infer that

$$
\begin{aligned}
\|y(t)\|_{X_{\gamma+\lambda}} & \leq M_{\alpha, \gamma+\lambda, T}(t-\varepsilon)^{\alpha-\gamma-\lambda}\|y(\varepsilon)\|_{X_{\alpha}}+\left\|\mathscr{I}_{S(\sigma \circ y)}(\varepsilon, t)\right\|_{X_{\gamma+\lambda}} \\
& \leq c_{1} M_{\alpha, \gamma+\lambda, T}(t-\varepsilon)^{\alpha-\gamma-\lambda} \\
& +\left\|\mathscr{I}_{S(\sigma \circ y)}\right\|_{C_{\eta+\alpha-\lambda-\gamma}\left([\varepsilon, T]_{<}^{2} ; X_{\lambda+\gamma}\right)}(t-\varepsilon)^{\eta+\alpha-\lambda-\gamma} \\
& \leq c_{6}(t-\varepsilon)^{\alpha-\gamma-\lambda} \varepsilon^{\lambda-\alpha}
\end{aligned}
$$

for every $t \in(\varepsilon, T]$ and some positive constant $c_{6}=c_{6}(\lambda, \gamma, \eta, \alpha, \sigma, x, \psi, \Re, T)$. In particular, since the range of the function $\varrho: D \rightarrow \mathbb{R}$, defined by $\varrho(\lambda, \gamma)=\lambda+\gamma$ for every $(\lambda, \gamma) \in D=\left\{(\lambda, \gamma) \in \mathbb{R}^{2}: \lambda \in[0, \eta+\alpha-1), \gamma \in[\alpha-\lambda, 1)\right\}$ is the interval $[\eta+\alpha-1, \eta+\alpha)$, for every $\mu \in[0, \eta+\alpha-1)$ we can choose $\lambda$ and $\gamma$ such that $1+\mu=\lambda+\gamma$. Then, from (3.34) we conclude that

$$
\|y(t)\|_{X_{1+\mu}} \leq c_{7}(t-\varepsilon)^{\alpha-1-\mu} \varepsilon^{\lambda-\alpha}, \quad t \in(\varepsilon, T],
$$

so that, for every $\varepsilon \in(0, T / 2)$,

$$
\|y(t)\|_{X_{1+\mu}} \leq c_{7} \varepsilon^{\lambda-1-\mu}, \quad t \in[2 \varepsilon, T],
$$

and $c_{7}=c_{7}(\lambda, \mu, \eta, \alpha, \sigma, x, \psi, \Re, T)$ is a positive constant, which depends in a continuous way on $\|x\|_{C^{\eta}([0, T])}$ and on $\mathfrak{R}$ but not on $\varepsilon$. From (3.35), estimate (3.4) follows at once. Finally, using again (3.33) and the smoothness properties of the semigroup $(S(t))_{t \geq 0}$, we conclude that $y \in E_{\eta+\alpha-\mu}\left([2 \varepsilon, T] ; X_{\mu}\right)$ for every $\mu \in$ $[\eta+\alpha-1, \eta+\alpha)$ and $\varepsilon \in(0, T / 2)$. 
Remark 3.1. (i) Theorem 3.1 generalizes the results in [9, Theorem 4.3].

(ii) From the last part of Step 3 in the proof of Theorem 3.1 it follows that $y \in$ $C\left((0, T] ; X_{\mu}\right)$ for any $\mu \in[0, \eta+\alpha)$.

(iii) In Step 3 of the proof of Theorem 3.1 we have proved that for each $r \in[\alpha, 1)$ there exists a constant $c$ such that

$$
\|y(t)\|_{X_{r}} \leq c t^{\alpha-r}, \quad t \in(0, T]
$$

for some constant $c$, independent of $t$. If $\psi \in X_{\gamma}$ for some $\gamma \in[\alpha, 1)$, then arguing as in estimate (3.27), we can easily show that we can replace $\alpha-r$ with $(\gamma-r) \wedge 0$ in (3.36), with $r \in[\alpha, 1)$. Based on this estimate, (3.28) and (3.29), we conclude that

$$
\begin{aligned}
\|y(t)-y(s)\|_{X} & \leq\left\|\left(\delta_{S} y\right)(t, s)\right\|_{X_{r}}+\|\mathfrak{a}(s, y) y(s)\|_{X_{r}} \\
& \leq c_{*}(t-s)^{\eta+\gamma-r}+c_{* *} S^{(\gamma-r-\beta) \wedge 0}|t-s|^{\beta}
\end{aligned}
$$

for every $\beta>0$ such that $r+\beta<1$, every $0<s<t \leq T$ and some positive constants $c_{*}$ and $c_{* *}$, independent of $s$ and $t$. Since $\beta<\eta+\gamma-r$, from (3.37) we conclude that

$$
\|y(t)-y(s)\|_{X_{r}} \leq c s^{(\gamma-r-\beta) \wedge 0}|t-s|^{\beta}, \quad 0<s<t \leq T .
$$

If $\gamma-r-\beta \geq 0$ then the above estimate can be extended to $s=0$. We will use these estimates in Sect. 5.

Remark 3.2. The result in Theorem 3.1 extend, using the same techniques, to the case of the Young equation

$$
\left\{\begin{array}{l}
\mathrm{d} y(t)=A y(t) \mathrm{d} t+\sum_{i=1}^{m} \sigma_{i}(y(t)) \mathrm{d} x_{i}(t), \quad t \in(0, T], \\
y(0)=\psi,
\end{array}\right.
$$

where the nonlinear terms $\sigma_{i}(i=1, \ldots, m)$ satisfy Hypotheses (3.1) and the paths $x_{i},(i=1, \ldots, m)$, belong to $C^{\eta}([0, T])$.

\subsection{The case when the semigroup has no smoothing effects}

The proof of Theorem 3.1 strongly relies on the smoothing effects on the semigroup $(S(t))_{t \geq 0}$, i.e., on condition 2.1(a), which in general is not satisfied when the semigroup associated with operator $A$ is merely strongly continuous. For instance, one may think to the semigroup of left-translations in the space of bounded and continuous functions over $\mathbb{R}^{d}$ or in the usual $L^{p}\left(\mathbb{R}^{d}\right)$-space related to the Lebesgue measure: the function $S(t) f$ has the same degree of smoothness as the function $f$.

In the proof of Theorem 3.1, condition (2.1)(a) is heavily used to prove that the mild solution $y$ to the nonlinear Young equation 3.2 takes values to $D(A)$.

In this subsection we show that partially removing condition (2.1)(a), i.e. assuming that it holds true only when $\alpha=\zeta$, and suitably choosing the intermediate spaces $X_{\alpha}$, the existence and uniqueness of a mild solution to Eq. (3.2) can still be guaranteed. 
Theorem 3.2. Let Hypotheses 2.1(i), 2.1(ii)(a) (with $\zeta=\alpha$ ), 2.1(ii)(b), 2.2 and 3.1 be satisfied, with $[a, b]=[0, T]$. Then, for every $\psi \in X_{\alpha}$, such that $\alpha \in(0,1 / 2)$ and $\eta+\alpha>1$, there exists a unique mild solution $y \in E_{\alpha}\left([0, T] ; X_{\alpha}\right)$ to Eq. (3.2).

Proof. The proof follows the same lines as the first two steps of the proof of Theorem 3.1. The only difference is that, under these weaker assumptions, Lemma 2.1 can be applied only with $r=k$, so that estimate (3.11) now reads as follows:

$$
\begin{aligned}
& \left\|\mathscr{I}_{S(\sigma \circ y)}\right\|_{C_{\eta}\left([a, b]_{<}^{2} ; X_{\alpha}\right)} \\
& \quad \leq C_{\alpha, \eta}\|x\|_{C^{\eta}([0, T])}\left(L_{\sigma}+L_{\sigma}^{\alpha}\right)\left(2+C_{\alpha, 0, T}\right)\left(1+\|y\|_{E_{\alpha}\left([a, b] ; X_{\alpha}\right)}\right) .
\end{aligned}
$$

From this point on the proof of the theorem carries on as in the proof of the quoted theorem.

We now provide an example of intermediate spaces $X_{\alpha}$ for which any strongly continuous semigroup satisfies Hypothesis 2.1(ii)(b) and 2.1(ii)(a), this latter at least with $\zeta=\alpha$.

Example 3.1. Let $A$ be the generator of a strongly continuous semigroup $(S(t))_{t \geq 0}$ and for each $\alpha \in(0,1)$ let us consider the Favard space

$$
F_{\alpha}=\left\{x \in X: \sup _{t \in(0,1]} \frac{\|S(t) x-x\|_{X}}{t^{\alpha}}<+\infty\right\},
$$

endowed with the norm

$$
\|x\|_{F_{\alpha}}=\sup _{t \in(0,1]} \frac{\|S(t) x-x\|_{X}}{t^{\alpha}}, \quad x \in F_{\alpha} .
$$

If $\alpha=k+\beta$ for some $k \in \mathbb{N}$ and $\beta \in(0,1)$, then

$$
F_{\alpha}=\left\{x \in D\left(A^{k}\right): \sup _{t \in(0,1]} \frac{\left\|S(t) A^{k} x-A^{k} x\right\|_{X}}{t^{\beta}}<+\infty\right\},
$$

endowed with the norm

$$
\|x\|_{F_{\alpha}}=\|x\|_{D\left(A^{k}\right)}+\left\|A^{k} x\right\|_{F_{\beta}}, \quad x \in F_{\alpha} .
$$

Each space $F_{\alpha}$ is a Banach space when endowed with the norm $\|\cdot\|_{F_{\alpha}}$.

Fix $\alpha \in \mathbb{R}, x \in F_{\alpha}$ and $t \in[0,+\infty)$. For any $s \in(0,1]$, we can estimate

$$
\frac{\|S(s) S(t) x-S(t) x\|_{X}}{s^{\alpha}} \leq\|S(t)\|_{\mathcal{L}(X)} \frac{\|S(s) x-x\|_{X}}{s^{\alpha}} \leq\|S(t)\| \mathcal{L}(X)\|x\|_{X_{\alpha}} .
$$

Hence, $S(t) x$ belongs to $F_{\alpha}$ and $\|S(t) x\|_{F_{\alpha}} \leq\|S(t)\|_{\mathcal{L}(X)}\|x\|_{F_{\alpha}}$, so that Hypothesis 2.1(ii)(a), with $\zeta=\alpha$ holds true if we take $X_{\alpha}=F_{\alpha}$. 
Let us prove that the semigroup $(S(t))_{t \geq 0}$ satisfies Hypothesis (2.1)(ii)(b) with $X_{\alpha}=$ $F_{\alpha}$. Fix $\mu, v \in(0,1]$ with $\mu>v, x \in X_{\mu}$ and $t \in(0,1]$. Then, for any $s \in(0, t]$ it holds that $s^{-v} \leq s^{-\mu} t^{\mu-v}$, so that

$$
\begin{aligned}
\left\|s^{-v}(S(s)-I)(S(t)-I) x\right\|_{X} & \leq\left\|s^{-v}(S(t+s) x-S(s) x-S(t) x+x)\right\|_{X} \\
& \leq\left\|s^{-\mu}(S(t+s) x-S(t) x)\right\|_{X} t^{\mu-v} \\
& +\left\|s^{-\mu}(S(s) x-x)\right\|_{X} t^{\mu-v} \\
& \leq\|S(t) x\|_{F_{\mu}} t^{\mu-v}+\|x\|_{F_{\mu}} t^{\mu-v} \\
& \leq\left(1+M_{0,0,1}\right) t^{\mu-v}\|x\|_{F_{\mu}} .
\end{aligned}
$$

On the other hand, if $s \in(t, 1]$, then $s^{-v}<t^{-v}$ so that

$$
\begin{aligned}
s^{-v}\|(S(s)-I)(S(t) x-x)\|_{X} & \leq\left\|t^{-\mu}(S(t+s) x-S(s) x)\right\|_{X} t^{\mu-v} \\
& +\left\|t^{-\mu}(S(t) x-x)\right\|_{X} t^{\mu-v} \\
& \leq\|S(s) x\|_{F_{\mu}} t^{\mu-v}+\|x\|_{F_{\mu}} t^{\mu-v} \\
& \leq\left(1+M_{0,0,1}\right) t^{\mu-v}\|x\|_{F_{\mu}} .
\end{aligned}
$$

We have so proved that

$$
\|S(t)-I\|_{\mathcal{L}\left(F_{\mu}, F_{v}\right)} \leq\left(1+M_{0,0,1}\right) t^{\mu-v}, \quad t \in(0,1] .
$$

and estimate (2.1)(ii)(b) follows, with $T=1$ and with $C_{\mu, v}=\left(1+M_{0,0,1}\right)$. If $T>1$ and $t \in(1, T]$, then

$$
\begin{aligned}
\sup _{s \in(0,1]} s^{-v}\|(S(s)-I)(S(t) x-x)\|_{X} & \leq\left(\|S(t)\|_{\mathcal{L}(X)}+1\right)\|x\|_{F_{\mu}} \\
& \leq\left(M_{0,0, T}+1\right)\|x\|_{F_{\mu}},
\end{aligned}
$$

so that estimate (2.1)(ii)(b) holds true in any interval $[0, T]$.

We refer the reader to [4, Chapter 2, Section 5.b] for further results on the Favard spaces.

Remark 3.3. Note that if $X=C_{b}(\mathbb{R})$ and $A$ is the first-order derivative, with $C_{b}^{1}(\mathbb{R})$ as domain, then $(S(t))_{t \geq 0}$ is the semigroup of left translations on $C_{b}(\mathbb{R})$. For every $\alpha \in(0,+\infty) \backslash \mathbb{N}, F_{\alpha}$ is the space of all functions $f: \mathbb{R} \rightarrow \mathbb{R}$, which are differentiable up to the $[\alpha]$-th order and such that the derivative of order $[\alpha]$ is bounded and $(\alpha-[\alpha])$ Hölder continuous on $\mathbb{R}$.

\section{The integral representation formula}

Knowing that mild solutions take their values in $D(A)$ we are in a position to prove that they solve equation (3.2) in a natural integral form. 
Definition 4.1. Let $y$ belong to $E_{\alpha}\left([0, T] ; X_{\alpha}\right) \cap L^{1}((0, T) ; D(A))$ for some $\alpha \in$ $(1-\eta, 1)$. We say that $y$ solves equation (3.2) in the integral form if, for every $t>0$, it satisfies the equation

$$
y(t)=\psi+\int_{0}^{t} A y(u) \mathrm{d} u+\int_{0}^{t} \sigma(y(u)) \mathrm{d} x(u), \quad t \in[0, T] .
$$

Remark 4.1. To prove that mild solutions verify (4.1), we first need to check that the integral

$$
\int_{0}^{t} \sigma(y(u)) \mathrm{d} x(u), \quad 0 \leq t \leq T
$$

is well defined as Young integral, when $y$ is the unique mild solution to (3.2). But, if $\sigma$ satisfies Hypothesis 3.1 , then for every $f \in E_{\alpha}\left([0, T] ; X_{\alpha}\right)$ and $x \in C^{\eta}([0, T])$, where $\eta \in(1 / 2,1)$ and $\alpha \in(1-\eta, 1)$, the Young integral

$$
\int_{s}^{t} \sigma(f(u)) \mathrm{d} x(u), \quad(s, t) \in[0, T]_{<}^{2},
$$

is well defined. Indeed, arguing as in the proof of (3.9) it can be easily checked that $\sigma \circ f \in C^{\alpha}([0, T] ; X)$. Therefore, Theorem 2.1 guarantees that the integral in (4.2) is well-defined.

We can now prove that, under Hypotheses 2.1, 2.2 and 3.1, the mild solution $y$ verifies (4.1) To prove this result, we first show that the mild solution to (3.2) can be approximated by mild solutions of classical problems.

Proposition 4.1. Let $\left(x_{n}\right) \subset C^{1}([0, T])$ be a sequence converging to $x$ in $C^{\eta}([0, T])$ for some $\eta>1 / 2$ and fix $\psi \in X_{\alpha}$ for some $\alpha \in(0,1 / 2)$ such that $\alpha+\eta>1$. For every $n \in \mathbb{N}$, denote by $y_{n}$ the mild solution to (3.2) with $x$ replaced by $x_{n}$, and let $y$ be the mild solution to (3.2). Then, the following properties are satisfied:

(i) $y_{n}$ converges to $y$ in $E_{\alpha}\left([0, T] ; X_{\alpha}\right)$ as $n$ tends to $+\infty$;

(ii) if we set $\mathbb{J}(t)=\int_{0}^{t} \sigma(y(u)) \mathrm{d} x(u)$ and $\mathbb{J}_{n}(t)=\int_{0}^{t} \sigma\left(y_{n}(u)\right) \mathrm{d} x_{n}(u)$ for every $t \in[0, T]$ and $n \in \mathbb{N}$, then $\mathbb{J}_{n}$ converges to $\mathbb{J}$ in $C^{\eta}([0, T] ; X)$ as $n$ tends to $+\infty$.

Proof. (i) We split the proof into two steps. In the first one, we show the assertion when $T$ is small enough and in the second step we remove this additional condition.

Step 1. Let us fix $\tau, \widetilde{T} \in[0, T]$ with $\tau<\widetilde{T}$. To begin with, we observe that applying Lemma 2.1 (with $k=r=\beta_{1}=\alpha, \beta=0$ and $a=\tau, b=\widetilde{T}$ ) and noticing that, by Corollary 2.1 (with $a=\tau$ and $b=\widetilde{T}),\left(\delta_{S} \mathscr{I}_{S f}(\tau, \cdot)\right)(s, t)=\mathscr{I}_{S f}(s, t)$ for every $(s, t) \in[\tau, \widetilde{T}]$, we can show that

$$
\begin{aligned}
\left\|\mathscr{I}_{S f}(\tau, \cdot)\right\|_{E_{\alpha}\left([\tau, \widetilde{T}] ; X_{\alpha}\right) \leq} & C(\widetilde{T}-\tau)^{\eta-\alpha}\left(\|f\|_{C\left([\tau, \widetilde{T}] ; X_{\alpha}\right)}\right. \\
& \left.+\left\|\delta_{S} f\right\|_{C_{\alpha}\left([\tau, \widetilde{T}]_{<}^{2} ; X\right)}\right)\|x\|_{C^{\eta}([0, T])}
\end{aligned}
$$


for every $x \in C^{\eta}([0, T])$ and $f \in E_{\alpha}([0, T] ; X) \cap C\left([0, T] ; X_{\alpha}\right)$ such that $\alpha \in$ $(0,1 / 2), \eta+\alpha>1$ and $\tau, \widetilde{T} \in[0, T]$, with $\tau<\widetilde{T}$, where $C=C_{\alpha, \eta, \alpha, \alpha}$ is the constant in Lemma 2.1.

Now, we fix $T_{*} \in(0, T]$ to be chosen later on. From (2.12) and (2.13), we get

$$
\begin{aligned}
y(t)-y_{n}(t) & =\int_{0}^{t} S(t-r) \sigma(y(r)) \mathrm{d} x(r)-\int_{0}^{t} S(t-r) \sigma\left(y_{n}(r)\right) \mathrm{d} x_{n}(r) \\
& =\int_{0}^{t} S(t-r) \sigma\left(y_{n}(r)\right) d \bar{x}_{n}(r) \\
& +\int_{0}^{t} S(t-r)\left(\sigma(y(r))-\sigma\left(y_{n}(r)\right)\right) \mathrm{d} x(r) \\
& =\mathbb{I}_{1, n}(t)+\mathbb{I}_{2, n}(t)
\end{aligned}
$$

for every $t \in\left[0, T_{*}\right]$, where $\bar{x}_{n}:=x-x_{n}$. Taking (3.10) and (3.7) into account, we can estimate

$$
\begin{aligned}
& \left\|\mathbb{I}_{1, n}\right\|_{E_{\alpha}\left(\left[0, T_{*}\right] ; X_{\alpha}\right)} \\
& \leq C T_{*}^{\eta-\alpha}\left(\left\|\sigma \circ y_{n}\right\|_{C\left(\left[0, T_{*}\right] ; X_{\alpha}\right)}+\left\|\delta_{S}\left(\sigma \circ y_{n}\right)\right\|_{C_{\alpha}\left(\left[0, T_{*}\right]_{<}^{2} ; X\right)}\right)\left\|\bar{x}_{n}\right\|_{C \eta([0, T])} \\
& \leq C T_{*}^{\eta-\alpha}\left(L_{\sigma}+L_{\sigma}^{\alpha}\right)\left(C_{\alpha, 0, T}+2\right)\left(1+\left\|y_{n}\right\|_{E_{\alpha}\left(\left[0, T_{*}\right] ; X_{\alpha}\right)}\right)\left\|\bar{x}_{n}\right\|_{C^{\eta}([0, T])},
\end{aligned}
$$

where $C$ is a positive constant which depends on $\alpha, \eta, \sigma$ and $T$. An inspection of the proof of estimate (3.6) shows that the constant $\mathfrak{R}$ depends in a continuous way on the $\eta$-Hölder norm of the path. Since $\sup _{n \in \mathbb{N}}\left\|x_{n}\right\|_{C^{\eta}([0, T])}<+\infty$, from (4.4) we can infer that

$$
\left\|\mathbb{I}_{1, n}\right\|_{E_{\alpha}\left(\left[0, T_{*}\right] ; X_{\alpha}\right)} \leq c T_{*}^{\eta-\alpha}\left(L_{\sigma}+L_{\sigma}^{\alpha}\right)\left(C_{\alpha, 0, T}+2\right)(1+\mathfrak{M})\left\|\bar{x}_{n}\right\|_{C^{\eta}([0, T])},
$$

for some positive constant $\mathfrak{M}$, independent of $n$. As far as $\mathbb{I}_{2, n}$ is considered, from (4.3), with $f$ replaced by $\sigma \circ y-\sigma \circ y_{n}$, and estimates (3.24), (3.25), we infer that

$$
\begin{aligned}
& \left\|\mathbb{I}_{2, n}\right\|_{E_{\alpha}\left(\left[0, T_{*}\right] ; X_{\alpha}\right)} \\
& \leq c T_{*}^{\eta-\alpha}\left(\left\|\sigma \circ y-\sigma \circ y_{n}\right\|_{C\left(\left[0, T_{*}\right] ; X_{\alpha}\right)}\right. \\
& \left.\quad+\left\|\delta_{S}\left(\sigma \circ y-\sigma \circ y_{n}\right)\right\|_{C_{\alpha}\left(\left[0, T_{*}\right]_{<}^{2} ; X\right)}\right)\|x\|_{C^{\eta}([0, T])} \\
& \leq \widetilde{c} T_{*}^{\eta-\alpha}\left\|y-y_{n}\right\|_{E_{\alpha}\left(\left[0, T_{*}\right] ; X_{\alpha}\right)}\|x\|_{C^{\eta}([0, T])},
\end{aligned}
$$

and $\widetilde{c}$ is a positive constant which depends on $\alpha, T, \sigma, \mathfrak{M}, K, \eta$ and on the constant $C_{\alpha, 0, T}$. We choose $T_{*} \leq T$ such that $\widetilde{c} T_{*}^{\eta-\alpha}\|x\|_{C^{\eta}([0, T])} \leq 1 / 2$ and use the previous estimate to conclude that

$$
\left\|y-y_{n}\right\|_{E_{\alpha}\left(\left[0, T_{*}\right] ; X_{\alpha}\right)} \leq 2 c T_{*}^{\eta-\alpha}\left(L_{\sigma}+L_{\sigma}^{\alpha}\right)\left(C_{\alpha, 0, T}+2\right)(1+\mathfrak{M})\left\|x_{n}-x\right\|_{C^{\eta}([0, T])}
$$

and, consequently, that $y_{n}$ converges to $y$ in $E_{\alpha}\left(\left[0, T_{*}\right] ; X_{\alpha}\right)$ as $n$ tends to $+\infty$. 
Step 2. If $T_{*}=T$ then we are done. Otherwise, let us fix $\widehat{T}:=\left(2 T_{*}\right) \wedge T$. For every $t \in\left[T_{*}, \widehat{T}\right]$, from $(3.5)$ we can write

$$
\begin{aligned}
y(t)-y_{n}(t)= & \int_{T_{*}}^{t} S(t-r)\left(\sigma(y(r))-\sigma\left(y_{n}(r)\right)\right) \mathrm{d} x(r) \\
& +\int_{T_{*}}^{t} S(t-r) \sigma\left(y_{n}(r)\right) d \bar{x}_{n}(r) \\
& +S\left(t-T_{*}\right)\left(y\left(T_{*}\right)-y_{n}\left(T_{*}\right)\right) .
\end{aligned}
$$

In Step 1 we have proved that $y_{n}\left(T_{*}\right)$ converges to $y\left(T_{*}\right)$ in $X_{\alpha}$ as $n$ tends to $+\infty$. Moreover, for every $(s, t) \in\left[T_{*}, T\right]_{<}^{2}$ it holds that $\delta_{S} S\left(\cdot-T_{*}\right)\left(y\left(T_{*}\right)-y_{n}\left(T_{*}\right)\right)(s, t)=0$. Hence, $\left\|S\left(\cdot-T_{*}\right)\left(y\left(T_{*}\right)-y_{n}\left(T_{*}\right)\right)\right\|_{E_{\alpha}\left(\left[T_{*}, \widehat{T}\right] ; X_{\alpha}\right)}$ vanishes as $n$ tends to $+\infty$. Repeating the same arguments as in Step 1, we conclude that

$$
\left\|y-y_{n}\right\|_{E_{\alpha}\left(\left[T_{*}, \widehat{T}\right] ; X_{\alpha}\right)} \leq 2 c T_{*}^{\eta-\alpha}\left(L_{\sigma}+L_{\sigma}^{\alpha}\right)\left(C_{\alpha, 0, T}+2\right)(1+\mathfrak{M})\left\|x_{n}-x\right\|_{C^{\eta}([0, T])},
$$

and therefore $y_{n}$ converges to $y$ in $E_{\alpha}\left(\left[T_{*}, \widehat{T}\right] ; X_{\alpha}\right)$ as $n$ tends to $+\infty$. If $\widehat{T}=T$ then the assertion follows. Otherwise by iterating this argument, we get the assertion in a finite number of steps.

(ii) As in the proof of property (i), we can write

$$
\begin{aligned}
\mathbb{J}_{n}(t)-\mathbb{J}(t) & =\int_{0}^{t} \sigma\left(y_{n}(u)\right) d \bar{x}_{n}(u)+\int_{0}^{t}\left(\sigma\left(y_{n}(u)\right)-\sigma(y(u))\right) \mathrm{d} x(u) \\
& =: \mathbb{J}_{1}^{n}(0, t)+\mathbb{J}_{2}^{n}(0, t) .
\end{aligned}
$$

From (2.5), (3.7) and (3.10), we infer that

$$
\begin{aligned}
&\left\|\mathbb{J}_{1}^{n}(0, t)\right\|_{X} \leq t^{\eta}\left(\left\|\sigma \circ y_{n}\right\|_{C([0, T] ; X)}+\frac{\left\|\sigma \circ y_{n}\right\|_{E_{\alpha}\left([0, T] ; X_{\alpha}\right)}}{1-2^{-(\eta+\alpha-1)}}\right)\left\|\bar{x}_{n}\right\|_{C^{\eta}([0, T])} \\
& \leq T^{\eta}\left(L_{\sigma}+\frac{\left(L_{\sigma}+L_{\sigma}^{\alpha}\right)\left(1+C_{\alpha, 0, T}\right)}{1-2^{-(\eta+\alpha-1)}}\right) \\
& \\
& \times\left(1+\sup _{n \in \mathbb{N}}\left\|y_{n}\right\|_{E_{\alpha}\left([0, T] ; X_{\alpha}\right)}\right)\left\|\bar{x}_{n}\right\|_{C^{\eta}([0, T])}
\end{aligned}
$$

for every $t \in[0, T]$, As far as the term $\mathbb{J}_{2}^{n}(0, t)$ is concerned, we argue similarly, taking advantage of the computations in (3.23) and estimate (3.24), and get

$$
\left\|\rrbracket_{2}^{n}(0, t)\right\|_{X} \leq C T^{\eta}\left\|y-y_{n}\right\|_{E_{\alpha}\left([0, T] ; X_{\alpha}\right)} .
$$

From (4.5) and (4.6) it thus follows that

$$
\sup _{t \in[0, T]}\left\|\mathbb{J}_{n}(t)-\mathbb{J}(t)\right\|_{X} \leq C^{\prime} T^{\eta}\left(\left\|x-x_{n}\right\|_{C^{\eta}([0, T])}+\left\|y-y_{n}\right\|_{E_{\alpha}\left([0, T] ; X_{\alpha}\right)}\right)
$$

for a suitable constant $C^{\prime}$, independent of $n$. From the assumptions on $x$ and $\left(x_{n}\right)$, and property (i), we conclude that $\mathbb{J}_{n}$ converges to $\mathbb{J}$ in $C([0, T] ; X)$ as $n$ tends to $+\infty$. 
To prove that $\mathbb{J}_{n}$ converges to $\mathbb{J}$ in $C^{\eta}([0, T] ; X)$, now it suffices to note that (see Remark 2.3)

$$
\left(\delta\left(\mathbb{J}_{n}-\mathbb{J}\right)\right)(s, t)=\mathbb{J}_{1}^{n}(s, t)+\mathbb{J}_{2}^{n}(s, t), \quad(s, t) \in[0, T]_{<}^{2} .
$$

and repeat the above computations to infer that

$$
\left[\mathbb{J}_{n}-\mathbb{J}_{C^{\eta}([0, T] ; X)} \leq C^{\prime}\left(\left\|x-x_{n}\right\|_{C^{\eta}([0, T])}+\left\|y-y_{n}\right\|_{E_{\alpha}\left([0, T] ; X_{\alpha}\right)}\right)\right.
$$

for every $n \in \mathbb{N}$.

We are now ready to show that the mild solution $y$ to (3.2) satisfies the integral representation formula (4.1).

Theorem 4.1. Let Hypotheses 2.1, 2.2 and 3.1 be satisfied and let $\psi \in X_{\alpha}$ for some $\alpha \in(0,1)$ such that $\alpha+\eta>1$. Further, let y be the unique mild solution to Eq. (3.2). Then, y satisfies (4.1).

Proof. Let $\left(x_{n}\right) \subset C^{1}([0, T])$ be a sequence of smooth paths which converges to $x$ in $C^{\eta}([0, T])$ as $n$ tends to $+\infty$. For every $n \in \mathbb{N}$, let $y_{n}$ be the unique mild solution to (3.2) with $x$ replaced by $x_{n}$. The computations in Step 3 of the proof of Theorem 3.1 with $x$ replaced by $x_{n}$ and the fact that $\sup _{n \in \mathbb{N}}\left\|x_{n}\right\|_{C^{\eta}([0, T])}<+\infty$ imply that $y_{n}(t)$ belongs to $D(A)$ for each $t \in(0, T]$ and $n \in \mathbb{N}$, and for every $\lambda \in[0, \eta+\alpha-1)$ there exists a positive constant $c=c(\lambda)$, independent of $n$, such that $\|A y(t)\|_{X} \leq c t^{\lambda-1}$ and $\left\|A y_{n}(t)\right\|_{X} \leq c t^{\lambda-1}$ for every $t \in(0, T]$ and $n \in \mathbb{N}$. From [12, Proposition 4.1.5] we infer that

$$
y_{n}(t)=\psi+\int_{0}^{t} A y_{n}(s) \mathrm{d} s+\int_{0}^{t} \sigma\left(y_{n}(s)\right) \mathrm{d} x_{n}(s), \quad t \in[0, T], \quad n \in \mathbb{N} .
$$

Let us fix $t \in(0, T]$. From Proposition 4.1 we know that $y_{n}$ converges to $y$ in $C([0, T] ; X)$ and $\int_{0}^{t} \sigma\left(y_{n}(s)\right) \mathrm{d} x_{n}(s)$ converges to $\int_{0}^{t} \sigma(y(s)) \mathrm{d} x(s)$ in $X$ as $n$ tends to $+\infty$. Hence, $\int_{0}^{t} y_{n}(s) \mathrm{d} s$ and

$$
A \int_{0}^{t} y_{n}(s) \mathrm{d} s=\int_{0}^{t} A y_{n}(s) \mathrm{d} s=y_{n}(t)-\psi-\int_{0}^{t} \sigma\left(y_{n}(s)\right) \mathrm{d} x_{n}(s)
$$

converge, as $n$ tends to $+\infty$, to $\int_{0}^{t} y(s) \mathrm{d} s$ and $y(t)-\psi-\int_{0}^{t} \sigma(y(s)) \mathrm{d} x(s)$, respectively, for every $t \in[0, T]$. Since $A$ is a closed operator, it follows that

$$
\int_{0}^{t} y(s) \mathrm{d} s \in D(A), \quad A \int_{0}^{t} y(s) \mathrm{d} s=y(t)-\psi-\int_{0}^{t} \sigma(y(s)) \mathrm{d} x(s) .
$$

Finally, since $\|A y(t)\|_{X} \leq c t^{\lambda-1}$ for every $t \in(0, T]$ (see (3.4) with $\mu=0$ ), we conclude that $A y$ belongs to $L^{1}((0, T) ; X)$. Hence, $A \int_{0}^{t} y(s) \mathrm{d} s=\int_{0}^{t} A y(s) \mathrm{d} s$, 
which gives

$$
y(t)=\psi+\int_{0}^{t} A y(s) \mathrm{d} s+\int_{0}^{t} \sigma(y(s)) \mathrm{d} x(s) .
$$

The arbitrariness of $t \in[0, T]$ yields the assertion.

Corollary 4.1. Let $\sigma_{i}: X \rightarrow X(i=1, \ldots, m)$ satisfy Hypotheses 3.1 and let the paths $x_{i}(i=1, \ldots, m)$ belong to $C^{\eta}([0, T])$. Then, the unique mild solution $y$ to (3.38) with $\psi \in X_{\alpha}$, with $\alpha+\eta>1$, satisfies the equation

$$
y(t)=\psi+\int_{0}^{t} A y(u) \mathrm{d} u+\sum_{i=1}^{m} \int_{0}^{t} \sigma_{i}(y(u)) \mathrm{d} x_{i}(u), \quad t \in[0, T] .
$$

Proof. The statement follows from Remark 3.2, and by repeating the computations in this section.

\section{Chain rule for nonlinear Young equations}

In this subsection we use the integral representation formula (4.1) of the unique mild solution $y$ to problem (3.2) to prove a chain rule for $F(\cdot, y(\cdot))$, where $F$ is a smooth function.

Theorem 5.1. Let $F \in C^{1}([0, T] \times X)$ be such that and $F_{x}$ is $\alpha$-Hölder continuous with respect to $t$, locally uniformly with respect to $x$, and is locally $\gamma$-Hölder continuous with respect to $x$, uniformly with respect to $t$, for some $\alpha, \gamma \in(0,1)$ such that $\eta+\alpha \gamma>$ 1. Further, let $y$ be the unique mild solution to (3.2). Then,

$$
\begin{aligned}
F(t, y(t))-F(s, y(s))= & \int_{s}^{t} F_{t}(u, y(u)) \mathrm{d} u+\int_{s}^{t}\left\langle F_{x}(u, y(u)), A y(u)\right\rangle \mathrm{d} u \\
& +\int_{s}^{t}\left\langle F_{x}(u, y(u)), \sigma(y(u))\right\rangle \mathrm{d} x(u)
\end{aligned}
$$

for every $(s, t) \in[0, T]$.

Proof. Let us fix $0<s<t \leq T$ and a sequence $\left(\Pi_{n}(s, t)\right)$ of partitions $\Pi_{n}(s, t)=$ $\left\{s=s_{0}^{n}<s_{1}^{n}<\ldots<s_{m_{n}}^{n}=t\right\}$ of $[s, t]$, with mesh-size which converges to zero, 
and note that

$$
\begin{aligned}
F( & , y(t))-F(s, y(s)) \\
= & \sum_{j=1}^{m_{n}} F\left(s_{j}^{n}, y\left(s_{j}^{n}\right)\right)-F\left(s_{j-1}^{n}, y\left(s_{j-1}^{n}\right)\right) \\
= & \sum_{j=1}^{m_{n}}\left[F\left(s_{j}^{n}, y\left(s_{j}^{n}\right)\right)-F\left(s_{j-1}^{n}, y\left(s_{j}^{n}\right)\right)+F\left(s_{j-1}^{n}, y\left(s_{j}^{n}\right)\right)-F\left(s_{j-1}^{n}, y\left(s_{j-1}^{n}\right)\right)\right] \\
= & \sum_{j=1}^{m_{n}} F_{t}\left(s_{j}^{n}, y\left(s_{j}^{n}\right)\right) \Delta s_{j}^{n}+\sum_{j=1}^{m_{n}}\left(F_{t}\left(\tilde{s}_{j}^{n}, y\left(s_{j}^{n}\right)\right)-F_{t}\left(s_{j}^{n}, y\left(s_{j}^{n}\right)\right)\right) \Delta s_{j}^{n} \\
& +\sum_{j=1}^{m_{n}}\left\langle F_{x}\left(s_{j-1}^{n}, y\left(s_{j-1}^{n}\right)\right), \Delta y_{j}\right\rangle+\sum_{j=1}^{m_{n}}\left\langle F_{x}\left(s_{j-1}^{n}, \tilde{y}_{j}\right)-F_{x}\left(s_{j-1}^{n}, y\left(s_{j-1}^{n}\right)\right), \Delta y_{j}\right\rangle \\
= & : I_{1, n}+I_{2, n}+I_{3, n}+I_{4, n},
\end{aligned}
$$

where $\Delta y_{j}=y\left(s_{j}^{n}\right)-y\left(s_{j-1}^{n}\right), \Delta s_{j}^{n}=s_{j}^{n}-s_{j-1}^{n}, \tilde{s}_{j}^{n}=s_{j-1}^{n}+\theta_{j}^{n}\left(s_{j}^{n}-s_{j-1}^{n}\right)$, $\tilde{y}_{j}=y\left(s_{j-1}^{n}\right)+\eta_{j}^{n}\left(y\left(s_{j}^{n}\right)-y\left(s_{j-1}^{n}\right)\right)$ and $\theta_{j}^{n}, \eta_{j}^{n} \in(0,1)$ are obtained from the mean-value theorem, for every $j=1, \ldots, m_{n}$.

Analysis of the terms $I_{1, n}$ and $I_{2, n}$. Since the function $s \mapsto F_{t}(s, y(s))$ is continuous in $[0, T], I_{1, n}$ converges to $\int_{s}^{t} F_{t}(u, y(u)) \mathrm{d} u$ as $n$ tends to $+\infty$. Moreover, since $y([0, T])$ is a compact subset of $X$, the restriction of function $F_{t}$ to $[0, T] \times y([0, T])$ is uniformly continuous. Thus, for every $\varepsilon>0$ there exists a positive constant $\delta$ such that $\left|F_{t}\left(t_{2}, x_{2}\right)-F_{t}\left(t_{1}, x_{1}\right)\right| \leq \varepsilon$ if $\left|t_{2}-t_{1}\right|^{2}+\left|x_{2}-x_{1}\right|^{2} \leq \delta^{2}$. As a byproduct, it follows that, if $|\Pi(s, t)| \leq \delta$, then $\left|I_{2, n}\right| \leq \varepsilon \sum_{j=1}^{n} \Delta s_{j}^{n}=\varepsilon(t-s)$ and this shows that $I_{2, n}$ converges to 0 as $n$ tends to $+\infty$.

Analysis of the term $I_{3, n}$. Using (4.1) we can write (see Remark 2.3)

$$
\begin{aligned}
\left\langle F_{x}\right. & \left.\left(s_{j-1}^{n}, y\left(s_{j-1}^{n}\right)\right), \Delta y_{j}\right\rangle \\
& =\left\langle F_{x}\left(s_{j-1}^{n}, y\left(s_{j-1}^{n}\right)\right), \int_{s_{j-1}^{n}}^{s_{j}^{n}} A y(u) d u+\int_{s_{j-1}^{n}}^{s_{j}^{n}} \sigma(y(u)) \mathrm{d} x(u)\right\rangle \\
& =\left\langle F_{x}\left(s_{j-1}^{n}, y\left(s_{j-1}^{n}\right)\right), A y\left(s_{j-1}^{n}\right)\right\rangle \Delta s_{j}^{n} \\
& +\left\langle F_{x}\left(s_{j-1}^{n}, y\left(s_{j-1}^{n}\right)\right), \int_{s_{j-1}^{n}}^{s_{j}^{n}}\left(A y(u)-A y\left(s_{j-1}^{n}\right)\right) d u\right\rangle \\
& +\left\langle F_{x}\left(s_{j-1}^{n}, y\left(s_{j-1}^{n}\right)\right), \int_{s_{j-1}^{n}}^{s_{j}^{n}}\left(\sigma(y(u))-\sigma\left(y\left(s_{j-1}^{n}\right)\right)\right) \mathrm{d} x(u)\right\rangle \\
& +\left\langle F_{x}\left(s_{j-1}^{n}, y\left(s_{j-1}^{n}\right)\right), \sigma\left(y\left(s_{j-1}^{n}\right)\right)\right\rangle\left(x\left(s_{j}^{n}\right)-x\left(s_{j-1}^{n}\right)\right)
\end{aligned}
$$

for $j=1, \ldots, m_{n}$. By assumptions, the function $s \mapsto F_{x}(s, y(s))$ is continuous with values in $X^{\prime}$. Similarly, by Theorem 3.1 the function $A y$ is continuous in $(0, T]$. Indeed, $y$ belongs to $E_{\eta+\alpha-\mu}\left([s, T] ; X_{\mu}\right)$ for every $\mu \in[\eta+\alpha-1, \eta+\alpha)$. Taking 
$\mu=1$ we deduce that $\left\|\left(\delta_{S} y\right)(u, w)\right\|_{X_{1}} \leq c|w-u|^{\eta+\alpha-1}$ for every $(u, w) \in[s, t]_{<}^{2}$ and some positive constant $c$, independent of $u$ and $w$. Hence,

$$
\begin{aligned}
\|A y(u)-A y(w)\|_{X} & \leq\left\|\left(\delta_{S} y\right)(u, w)\right\|_{X_{1}}+\|\mathfrak{a}(u, w) A y(u)\|_{X} \\
& \leq c|w-u|^{\eta+\alpha-1}+\|\mathfrak{a}(u, w) A y(u)\|_{X} .
\end{aligned}
$$

Choosing $\mu=1+\rho$ for some $\rho<\eta+\alpha-1$ and using (2.1)(b) we get

$$
\|\mathfrak{a}(u, w) A y(w)\|_{X} \leq C_{0, \rho}|w-u|^{\rho}\|A y(w)\|_{X_{\rho}} \leq C_{0, \rho}|w-u|^{\rho}\|y\|_{C\left([\varepsilon, T] ; X_{1+\rho}\right)}
$$

Therefore, $A y$ is $\rho$-Hölder continuous in $[\varepsilon, T]$ for any $\varepsilon \in(0, T)$. Since $s>0$, it follows that $u \mapsto A y(u)$ is continuous in $[s, T]$, and we thus conclude that

$$
\lim _{n \rightarrow+\infty} \sum_{j=1}^{m_{n}}\left\langle F_{x}\left(s_{j-1}^{n}, y\left(s_{j-1}^{n}\right)\right), A y\left(s_{j-1}^{n}\right)\right\rangle \Delta s_{j}^{n}=\int_{s}^{t}\left\langle F_{x}(u, y(u)), A y(u)\right\rangle d u
$$

and

$$
\begin{aligned}
\left\|\int_{s_{j-1}^{n}}^{s_{j}^{n}}\left(A y(u)-A y\left(s_{j-1}^{n}\right)\right) d u\right\|_{X} & \leq[A y]_{C^{\rho}([s, T] ; X)}\left|s_{j}^{n}-s_{j-1}^{n}\right|^{1+\rho} \\
& \leq[A y]_{C^{\rho}([s, T] ; X)}\left(s_{j}^{n}-s_{j-1}^{n}\right)\left|\Pi_{n}(s, t)\right|^{\rho}
\end{aligned}
$$

for every $j=1, \ldots, m_{n}$, so that

$$
\begin{aligned}
& \left|\sum_{j=1}^{m_{n}}\left\langle F_{x}\left(s_{j-1}^{n}, y\left(s_{j-1}^{n}\right)\right), \int_{s_{j-1}^{n}}^{s_{j}^{n}}\left(A y(u)-A y\left(s_{j-1}^{n}\right)\right) d u\right\rangle\right| \\
& \quad \leq\left\|F_{x}\right\|_{C\left([0, T] \times y([0, T]) ; X^{\prime}\right)}[A y]_{C^{\rho}([s, T] ; X)}(t-s)\left|\Pi_{n}(s, t)\right|^{\rho}
\end{aligned}
$$

and the right-hand side of the previous inequality vanishes as $n$ tends to $+\infty$.

Let us consider the third term in the right-hand side of (5.1). From Theorem 2.1 and recalling that $\alpha+\eta>1$, we infer that

$$
\begin{aligned}
& \left\|\int_{s_{j-1}^{n}}^{s_{j}^{n}}\left(\sigma(y(u))-\sigma\left(y\left(s_{j-1}^{n}\right)\right)\right) \mathrm{d} x(u)\right\|_{X} \\
& \quad=\left\|\int_{s_{j-1}^{n}}^{s_{j}^{n}} \sigma(y(u)) \mathrm{d} x(u)-\sigma\left(y\left(s_{j-1}^{n}\right)\right)\left(x\left(s_{j}^{n}\right)-x\left(s_{j-1}^{n}\right)\right)\right\|_{X} \\
& \quad \leq \frac{1}{1-2^{\alpha-\eta-1}}\|\delta(\sigma \circ y)\|_{C_{\alpha}\left([0, T]_{<}^{2} ; X\right)}\|x\|_{C^{\eta}([0, T])}\left|s_{j}^{n}-s_{j-1}^{n}\right|^{\alpha+\eta} \\
& \quad \leq \frac{1}{1-2^{\alpha-\eta-1}}\|\delta(\sigma \circ y)\|_{C_{\alpha}\left([0, T]_{<}^{2} ; X\right)}\|x\|_{C^{\eta}([0, T])}\left(s_{j}^{n}-s_{j-1}^{n}\right)\left|\Pi_{n}(s, t)\right|^{\alpha+\eta-1}
\end{aligned}
$$


for $j=1, \ldots, m_{n}$. Hence,

$$
\begin{aligned}
& \left|\sum_{j=1}^{m_{n}}\left\langle F_{x}\left(s_{j-1}^{n}, y\left(s_{j-1}^{n}\right)\right), \int_{s_{j-1}^{n}}^{s_{j}^{n}}\left(\sigma(y(u))-\sigma\left(y\left(s_{j-1}^{n}\right)\right)\right) \mathrm{d} x(u)\right\rangle\right| \\
\leq & \frac{1}{1-2^{\alpha-\eta-1}}\left\|F_{x}\right\|_{C\left([0, T] \times y([0, T]) ; X^{\prime}\right)}(t-s)\left|\Pi_{n}(s, t)\right|^{\alpha+\eta-1} .
\end{aligned}
$$

Letting $n$ tend to $+\infty$ gives

$$
\lim _{n \rightarrow+\infty} \sum_{j=1}^{m_{n}}\left\langle F_{x}\left(s_{j-1}^{n}, y\left(s_{j-1}^{n}\right)\right), \int_{s_{j-1}^{n}}^{s_{j}^{n}}\left(\sigma(y(u))-\sigma\left(y\left(s_{j-1}^{n}\right)\right)\right) \mathrm{d} x(u)\right\rangle=0 .
$$

To conclude the study of $I_{3, n}$ it remains to consider the term

$$
\left\langle F_{x}\left(s_{j-1}^{n}, y\left(s_{j-1}^{n}\right)\right), \sigma\left(y\left(s_{j-1}^{n}\right)\right)\right\rangle\left(x\left(s_{j}^{n}\right)-x\left(s_{j-1}^{n}\right)\right) .
$$

For this purpose, we introduce the function $g:[s, t] \rightarrow \mathbb{R}$, defined by $g(\tau)=$ $\left\langle F_{x}(\tau, y(\tau)), \sigma(y(\tau))\right\rangle$ for every $\tau \in[s, t]$. Let us prove that $g \in C^{\alpha \gamma}([s, t])$. To this aim, we recall that

$$
\|\sigma(y(\tau))\|_{X} \leq K_{0, \alpha}\|\sigma(y(\tau))\|_{X_{\alpha}} \leq K_{0, \alpha} L_{\sigma}^{\alpha}\left(1+\|y\|_{C\left([0, T] ; X_{\alpha}\right)}\right), \quad \tau \in[0, T] .
$$

Hence, we can estimate

$$
\begin{aligned}
& \left|g\left(\tau_{2}\right)-g\left(\tau_{1}\right)\right| \\
& \quad=\left|\left\langle F_{x}\left(\tau_{2}, y\left(\tau_{2}\right)\right), \sigma\left(y\left(\tau_{2}\right)\right)\right\rangle-\left\langle F_{x}\left(\tau_{1}, y\left(\tau_{1}\right)\right), \sigma\left(y\left(\tau_{1}\right)\right)\right\rangle\right| \\
& \quad \leq\left|\left\langle F_{x}\left(\tau_{2}, y\left(\tau_{2}\right)\right)-F_{x}\left(\tau_{2}, y\left(\tau_{1}\right)\right), \sigma\left(y\left(\tau_{2}\right)\right)\right\rangle\right| \\
& \quad+\left\langle F_{x}\left(\tau_{2}, y\left(\tau_{1}\right)\right)-F_{x}\left(\tau_{1}, y\left(\tau_{1}\right)\right), \sigma\left(y\left(\tau_{2}\right)\right)\right\rangle \\
& \quad+\left|\left\langle F_{x}\left(\tau_{1}, y\left(\tau_{1}\right)\right), \sigma\left(y\left(\tau_{2}\right)\right)-\sigma\left(y\left(\tau_{1}\right)\right)\right\rangle\right| \\
& \quad \leq K_{0, \alpha} L_{\sigma}^{\alpha} \sup _{t \in[0, T]}\left\|F_{x}(t, \cdot)\right\|_{C^{\gamma}\left(y([0, T]) ; X^{\prime}\right)}\left(1+\|y\|_{C\left([0, T] ; X_{\alpha}\right)}\right)\left\|y\left(\tau_{2}\right)-y\left(\tau_{1}\right)\right\|_{X}^{\gamma} \\
& \quad+K_{0, \alpha} L_{\sigma}^{\alpha} \sup _{x \in y([0, T])}\left[F_{x}(\cdot, x)\right]_{C^{\alpha}\left([0, T] ; X^{\prime}\right)}\left(1+\|y\|_{C\left([0, T] ; X_{\alpha}\right)}\right)\left|\tau_{2}-\tau_{1}\right|^{\alpha} \\
& \quad+L_{\sigma}\left\|F_{x}\right\|_{C\left([0, T] \times y([0, T]) ; X^{\prime}\right)}\left\|y\left(\tau_{2}\right)-y\left(\tau_{1}\right)\right\| X \\
& \quad \leq\left(K_{0, \alpha} L_{\sigma}^{\alpha} \sup _{t \in[0, T]}\left\|F_{x}(t, \cdot)\right\|_{C \gamma\left(y([0, T]) ; X^{\prime}\right)}\left(1+\|y\|_{C\left([0, T] ; X_{\alpha}\right)}\right)[y]_{C^{\alpha}([0, T] ; X)}\right. \\
& \quad+K_{0, \alpha} L_{\sigma}^{\alpha} \sup _{x \in y([0, T])}\left[F_{x}(\cdot, x)\right]_{C^{\alpha}\left([0, T] ; X^{\prime}\right)}\left(1+\|y\|_{C\left([0, T] ; X_{\alpha}\right)}\right) T^{\alpha(1-\gamma)} \\
& \left.\quad+L_{\sigma}\left\|F_{x}\right\|_{C\left([0, T] \times y([0, T]) ; X^{\prime}\right)}[y]_{C^{\alpha}([0, T] ; X)} T^{\alpha(1-\gamma)}\right)\left|\tau_{2}-\tau_{1}\right|^{\alpha \gamma},
\end{aligned}
$$


for every $\tau_{1}, \tau_{2} \in[s, t]$, which shows that $g$ is $\alpha \gamma$-Hölder continuous in $[s, t]$. Since $\eta+\gamma \alpha>1$, we can apply Theorem 2.1 which implies that

$$
\begin{aligned}
& \lim _{n \rightarrow+\infty} \sum_{j=1}^{m_{n}}\left\langle F_{x}\left(s_{j-1}^{n}, y\left(s_{j-1}^{n}\right)\right), \sigma\left(y\left(s_{j-1}^{n}\right)\right)\right\rangle\left(x\left(s_{j}^{n}\right)-x\left(s_{j-1}^{n}\right)\right) \\
& =\int_{s}^{t}\left\langle F_{x}(u, y(u)), \sigma(y(u))\right\rangle \mathrm{d} x(u),
\end{aligned}
$$

where the integral is well-defined as Young integral. From (5.2)-(5.5) we conclude that

$$
\lim _{n \rightarrow+\infty} I_{3, n}=\int_{s}^{t}\left\langle F_{x}(u, y(u)), A y(u)\right\rangle d u+\int_{s}^{t}\left\langle F_{x}(u, y(u)), \sigma(y(u))\right\rangle \mathrm{d} x(u) .
$$

To complete the proof, we observe that $I_{4, n}$ converges to 0 as $n$ tends to $+\infty$. This property can be checked arguing as we did for the term $I_{2, n}$, noting that $F_{x}$ is uniformly continuous in $[0, T] \times y([0, T])$.

Summing up, we have proved that

$$
\begin{aligned}
F(t, y(t))-F(s, y(s))= & \int_{s}^{t} F_{t}(u, y(u)) d u+\int_{s}^{t}\left\langle F_{x}(u, y(u)), A y(u)\right\rangle d u \\
& +\int_{s}^{t}\left\langle F_{x}(u, y(u)), \sigma(y(u))\right\rangle \mathrm{d} x(u),
\end{aligned}
$$

for every $0<s<t \leq T$. As $s$ tends to $0^{+}$, the left-hand side of (5.6) converges to $F(t, y(t))-F(0, y(0))$. As far as the right-hand side is concerned, the first and the third term converge to the corresponding integrals over $[0, t]$ since the functions $u \mapsto F_{t}(u, y(u))$ and $u \mapsto F_{x}(u, y(u))$ are continuous in [0,T]. As far as the second term in the right-hand side of (5.6) is concerned, thanks to (3.4) with $\mu=0$ we can apply the dominated convergence theorem which yields the convergence to the integral over $(0, t)$. The assertion in its full generality follows.

The same arguments as in the proof of Theorem 5.1 and Corollary 4.1 give the following result.

Corollary 5.1. Let $\sigma_{i}: X \rightarrow X(i=1, \ldots, m)$ satisfy Hypotheses 3.1, let the paths $x_{i} \in C^{\eta}([0, T])(i=1, \ldots, n)$ belong to $C^{\eta}([0, T])$, and let $y$ be the unique mild solution to (3.38) with $\psi \in X_{\alpha}$, with $\alpha+\eta>1$. Then, for any function $F$ satisfying the assumptions in Theorem 5.1 it holds that

$$
\begin{aligned}
F(t, y(t))-F(s, y(s))= & \int_{s}^{t} F_{t}(u, y(u)) d u+\int_{s}^{t}\left\langle F_{x}(u, y(u)), A y(u)\right\rangle d u \\
& +\sum_{i=1}^{m} \int_{s}^{t}\left\langle F_{x}(u, y(u)), \sigma_{i}(y(u))\right\rangle \mathrm{d} x_{i}(u)
\end{aligned}
$$

for every $(s, t) \in[0, T]$. 
As an immediate application of the chain rule, we provide necessary conditions, in the contexts of Hilbert spaces, for the invariance of the set $K=\{x \in X:\langle x, \varphi\rangle \leq 0\}$ for the mild solution $y$ to (3.38), where invariance means that, if $\psi \in X_{\alpha} \cap K$, then $y(t)$ belongs to $K$ for any $t \in[0, T]$. For this purpose, we assume that $A: D(A) \subset X \rightarrow X$ is a self-adjoint nonpositive closed operator which generates an analytic semigroup of bounded linear operators $(S(t))_{t \geq 0}$ on $H$ and that the results so far proved hold true with $X_{\zeta}=D\left((-A)^{\zeta}\right)$ for any $\zeta \geq 0$.

Proposition 5.1. Let Hypotheses 2.1, 2.2, 3.1 be fulfilled with $\eta+\alpha>1$. Let $\varphi \in X_{\varepsilon}$ for some $\varepsilon \in[0,1), \psi \in X_{\zeta}$ for some $\zeta \in[\alpha, 1)$ and let $K:=\{x \in X:\langle x, \varphi\rangle \leq 0\}$ be invariant for $y$. The following properties are satisfied.

(i) If $\psi \in \partial K$ and $\eta \leq \zeta+\varepsilon \leq 1$, then

$$
\limsup _{t \rightarrow 0^{+}} t^{-\beta} \sum_{i=1}^{m}\left\langle\varphi, \sigma_{i}(\psi)\right\rangle\left(x_{i}(t)-x_{i}(0)\right) \leq 0,
$$

for $\beta \in[\eta, \zeta+\varepsilon)$ and

$$
\begin{gathered}
\sup _{\lambda>0} \limsup _{t \rightarrow 0^{+}} t^{-\beta}\left(-\int_{0}^{t} \frac{(\lambda+\langle\varphi, y(s)\rangle)_{+}}{\lambda}\left\langle(-A)^{\varepsilon} \varphi,(-A)^{1-\varepsilon} y(s)\right\rangle \mathrm{d} s\right. \\
\left.+\sum_{i=1}^{m}\left\langle\varphi, \sigma_{i}(\psi)\right\rangle\left(x_{i}(t)-x_{i}(0)\right)\right) \leq 0
\end{gathered}
$$

for $\beta \in[\zeta+\varepsilon, 1]$.

(ii) If $y\left(t_{0}\right) \in \partial K$ for some $t_{0} \in[0, T)$ and $\zeta+\varepsilon>1$, then

$$
\limsup _{t \rightarrow t_{0}^{+}}\left|t-t_{0}\right|^{-\beta} \sum_{i=1}^{m}\left\langle\varphi, \sigma_{i}\left(y\left(t_{0}\right)\right)\right\rangle\left(x_{i}(t)-x_{i}\left(t_{0}\right)\right) \leq 0,
$$

if $\beta \in[\eta, 1)$ and

$$
\begin{aligned}
& \limsup _{t \rightarrow t_{0}^{+}}\left|t-t_{0}\right|^{-1} \sum_{i=1}^{m}\left\langle\varphi, \sigma_{i}\left(y\left(t_{0}\right)\right)\right\rangle\left(x_{i}(t)-x_{i}\left(t_{0}\right)\right) \\
& -\left\langle(-A)^{\varepsilon} \varphi,(-A)^{1-\varepsilon} y\left(t_{0}\right)\right\rangle \leq 0,
\end{aligned}
$$

if $\beta=1$.

Remark 5.1. If $t_{0}>0$ in (ii) then $y(t) \in X_{1+\mu}$ for any $\mu \in[0, \eta+\alpha-1)$ and $t \in(0, T]$ (see Theorem 3.1). Hence, the condition $\zeta+\varepsilon>1$ is automatically satisfied.

Proof. For any $\lambda>0$ we introduce the function $F_{\lambda}: X \rightarrow X$, defined by $F_{\lambda}(x):=$ $(\lambda+\langle\varphi, x\rangle)_{+}^{2}$, for any $x \in X$. As it is easily seen, each function $F_{\lambda}$ belongs to $C^{1,1}(X)$ and $D F_{\lambda}(x)=2(\lambda+\langle\varphi, x\rangle)_{+} \varphi$ for any $x \in X$. Further, for any $x \in K$ it holds that 
$F_{\lambda}(x) \leq \lambda^{2}$, and $F_{\lambda}(x)=\lambda^{2}$ if and only if $x \in \partial K$. Fix $t_{0} \in[0, T)$. If $y\left(t_{0}\right) \in \partial K$ then from (5.7) it follows that

$$
\begin{aligned}
0 \geq & F_{\lambda}(y(t))-F_{\lambda}\left(y\left(t_{0}\right)\right) \\
= & -2 \int_{t_{0}}^{t}(\lambda+\langle\varphi, y(s)\rangle)_{+}\left\langle(-A)^{\varepsilon} \varphi,(-A)^{1-\varepsilon} y(s)\right\rangle \mathrm{d} s \\
& +2 \sum_{i=1}^{m} \lambda\left\langle\varphi, \sigma_{i}\left(y\left(t_{0}\right)\right)\right\rangle\left(x_{i}(t)-x_{i}\left(t_{0}\right)\right)+\mathscr{R}_{f}\left(t_{0}, t\right)
\end{aligned}
$$

for any $t \in\left[t_{0}, T\right]$ and any $\lambda>0$, where in the equality we have used (2.3) with $f(t)=(\lambda+\langle\varphi, y(t)\rangle)_{+}\left\langle\varphi, \sigma_{i}(y(t))\right\rangle$ for $t \in[0, T]$. We recall that from (2.4) it follows that $\mathscr{R}_{f}\left(t_{0}, t\right)=o\left(\left|t-t_{0}\right|\right)$ as $t \rightarrow t_{0}^{+}$, and from Remark 3.1(ii) we know that $y \in C\left((0, T] ; X_{\mu}\right)$ for any $\mu \in[0, \eta+\alpha)$.

Now, we separately consider the cases $(i)$ and $(i i)$.

(i). Fix $\psi \in \partial K \cap X_{\zeta}$ with $\eta \leq \zeta+\varepsilon \leq 1$. From (3.36) with $r=1-\varepsilon$ we infer that there exists a positive constant $c$, independent of $s$, such that

$$
\left|(\lambda+\langle\varphi, y(s)\rangle)_{+}\left\langle(-A)^{\varepsilon} \varphi,(-A)^{1-\varepsilon} y(s)\right\rangle\right| \leq \lambda c s^{\zeta+\varepsilon-1}, \quad s \in(0, T] .
$$

Let $\beta \in[\eta, 1]$. Dividing by $t^{\beta}$ and $\lambda$ both sides of (5.8) (with $t_{0}=0$ ) and taking (5.9) into account, the assertion follows easily.

(ii). Fix $t_{0} \in[0, T)$ with $y\left(t_{0}\right) \in \partial K$ and $\zeta+\varepsilon>1$. Since $1-\varepsilon<\zeta, y$ is continuous up to 0 with values in $X_{1-\varepsilon}$. Indeed, from $(2.1)(b)$ we get $\| S(t) \psi-$ $\psi \|_{X_{1-\varepsilon}} \leq C t^{\zeta+\varepsilon-1}$ for every $t \in[0, T]$, and from (3.3) and the smoothness of $\mathscr{I}_{S(\sigma \circ y)}$ at $t=0$ we infer that $y \in C_{b}\left([0, T] ; X_{1-\varepsilon}\right)$. As a consequence, the function $s \mapsto(\lambda+\langle\varphi, y(s)\rangle)_{+}\left\langle(-A)^{\varepsilon} \varphi,(-A)^{1-\varepsilon} y(s)\right\rangle$ belongs to $C_{b}([0, T])$. Let $\beta \in[\eta, 1]$. Dividing (5.8) by $\left|t-t_{0}\right|^{\beta}$ and $\lambda$, and letting $t \rightarrow t_{0}^{+}$, the assertion follows also in this case.

\section{Examples}

In this section, we provide two examples to which our results apply. We consider the second-order elliptic operator $\mathcal{A}$, defined by

$$
\mathcal{A}=\sum_{i, j=1}^{d} q_{i j} D_{i j}+\sum_{j=1}^{d} b_{j} D_{j}+c .
$$

Example 6.1. Let us assume that the coefficients of the operator $\mathcal{A}$ are bounded and $\beta$-Hölder continuous on $\mathbb{R}^{d}$, for some $\beta \in(0,1)$, and $\sum_{i, j=1}^{d} q_{i j}(x) \xi_{i} \xi_{j} \geq \mu|\xi|^{2}$ for every $x, \xi \in \mathbb{R}^{d}$ and some positive constant $\mu$. 
Let $A$ be the realization of operator $\mathcal{A}$ in $X=C_{b}\left(\mathbb{R}^{d}\right)$ with domain

$$
D(A)=\left\{u \in C_{b}\left(\mathbb{R}^{d}\right) \cap \bigcap_{p<+\infty} W_{\mathrm{loc}}^{2, p}\left(\mathbb{R}^{d}\right): \mathcal{A} u \in C_{b}\left(\mathbb{R}^{d}\right)\right\} .
$$

For every $\alpha \in(0,2) \backslash\{1 / 2,1\}$, we take $X_{\alpha}=C_{b}^{2 \alpha}\left(\mathbb{R}^{d}\right)$ endowed with the classical norm of $C_{b}^{2 \alpha}\left(\mathbb{R}^{d}\right)$. Moreover, we take as $X_{1 / 2}$ the Zygmund space of all bounded functions $g: \mathbb{R}^{d} \rightarrow \mathbb{R}$ such that

$$
[g]_{X^{1 / 2}}=\sup _{x \neq y} \frac{\left|g(x)+g(y)-2 g\left(2^{-1}(x+y)\right)\right|}{|x-y|}<+\infty,
$$

endowed with the norm $\|g\|_{X_{1 / 2}}=\|g\|_{\infty}+[g]_{X^{1 / 2}}$. It is well known that $A$ generates an analytic semigroup on $C_{b}\left(\mathbb{R}^{d}\right)$ and $X_{\alpha}$ is the interpolation space of order $\alpha$ between $X$ and $X_{1}=D(A)$. We refer the reader to e.g., [11, Chapters 3 and 14]. Finally, we fix a function $\hat{\sigma} \in C_{b}^{2}(\mathbb{R})$ and note that the function $\sigma: X \rightarrow X$, defined by $\sigma(f)=\hat{\sigma} \circ f$

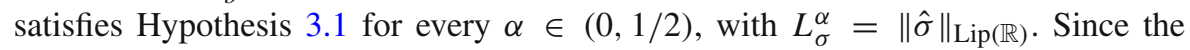
assumptions of Theorem 3.1 are satisfied, we conclude that, for every $\psi \in C_{b}^{\alpha}\left(\mathbb{R}^{d}\right)$ $(\alpha \in(0,1))$, there exists a unique solution $y$ to Problem (3.2), which takes values in $D(A)$.

Example 6.2. Let $\Omega \subset \mathbb{R}^{n}$ be a bounded open domain with $C^{2}$-boundary and assume that the coefficients $q_{i j}(i, j=1, \ldots, d)$ of operator $\mathcal{A}$ are uniformly continuous in $\Omega$, whereas the other coefficients are in $L^{\infty}(\Omega)$. We further assume that $\sum_{i, j=1}^{d} q_{i j}(x) \xi_{i} \xi_{j} \geq \mu|\xi|^{2}$ for every $x, \xi \in \mathbb{R}^{d}$ and some positive constant $\mu$ and, for $p \in(1,+\infty)$, we denote by $A_{p}$ the realization of the operator $\mathcal{A}$ in $X=L^{p}(\Omega)$ with homogeneous Dirichlet boundary conditions, with domain $D\left(A_{p}\right)=W^{2, p}(\Omega) \cap$ $W_{0}^{1, p}(\Omega)$. It is well-known that $A_{p}$ generates an analytic semigroup on $L^{p}(\Omega)$. We assume that $p>n$. Hence, $n /(2 p)<1 / 2$. It is also well-known that, for any $\alpha \in(1 /(2 p), 1)$,

$$
D_{A_{p}}(\alpha, p)=\left(L^{p}(\Omega), D\left(A_{p}\right)\right)_{\alpha, p}=W_{0}^{2 \alpha, p}(\Omega),
$$

where with $W_{0}^{2 \alpha, p}(\Omega)$ we denote the fractional Sobolev space of order $2 \alpha$ with null trace on $\partial \Omega$. We set $X_{\alpha}:=W_{0}^{2 \alpha, p}(\Omega)$.

Let us fix $f \in C_{b}^{2}(\mathbb{R})$ with $f(0)=0$ and let us define the function $\sigma$ by setting $\sigma \circ y(\cdot)=f(y(\cdot))$ for any $y \in L^{p}(\Omega)$. It is not hard to show that $\sigma: X \rightarrow X$ is Fréchet differentiable with bounded and locally Lipschitz Fréchet derivative. We claim that for $\alpha \in(n /(2 p), 1 / 2)$ the function $\sigma$ satisfies condition (3.1) and it is locally Lipschitz continuous in $X_{\alpha}$. Let us notice that $\sigma \circ y \in W_{0}^{2 \alpha, p}(\Omega)$ for any $y \in W_{0}^{2 \alpha, p}(\Omega)$. Let us denote by $\sigma^{\prime}$ the Fréchet derivative of $\sigma$. For any $y, h \in X_{\alpha}$ it holds that $\left(\left(\sigma^{\prime} \circ y\right) h\right)(\xi)=f^{\prime}(y(\xi)) h(\xi)$ for almost every $\xi \in \Omega$. Hence,

$$
\left\|\left(\sigma^{\prime} \circ y\right) h\right\|_{L^{p}(\Omega)} \leq\left\|f^{\prime}\right\|_{\infty}\|h\|_{L^{p}(\Omega)} \leq\|f\|_{C_{b}^{2}(\mathbb{R})}\|h\|_{X_{\alpha}} .
$$


Further, we recall that since $2 \alpha p>n$, it follows that $W^{2 \alpha, p}(\Omega) \subset C(\bar{\Omega})$. Therefore,

$$
\begin{aligned}
{\left[\left(\sigma^{\prime} \circ y\right) h\right]_{W^{2 \alpha, p}(\Omega)} } & =\int_{\Omega \times \Omega} \frac{\left|f^{\prime}(y(\xi)) h(\xi)-f^{\prime}(y(\eta)) h(\eta)\right|^{p}}{|\xi-\eta|^{2 \alpha p+n}} \mathrm{~d} \xi \mathrm{d} \eta \\
& \leq \int_{\Omega \times \Omega} \frac{\left|f^{\prime}(y(\xi)) h(\xi)-f^{\prime}(y(\xi)) h(\eta)\right|^{p}}{|\xi-\eta|^{2 \alpha p+n}} \mathrm{~d} \xi \mathrm{d} \eta \\
& +\int_{\Omega \times \Omega} \frac{\left|f^{\prime}(y(\xi)) h(\eta)-f^{\prime}(y(\eta)) h(\eta)\right|^{p}}{|\xi-\eta|^{2 \alpha p+n}} \mathrm{~d} \xi \mathrm{d} \eta \\
& \leq\|f\|_{C_{b}^{2}(\mathbb{R})}^{p}[h]_{W^{2 \alpha, p}(\Omega)}^{p}+\|f\|_{C_{b}^{2}(\mathbb{R})}^{p}\|h\|_{\infty}^{p}[y]_{W^{2 \alpha, p}(\Omega)}^{p} \\
& \leq c_{1}^{p}\|h\|_{X_{\alpha}}^{p}\left(1+\|y\|_{X_{\alpha}}^{p}\right),
\end{aligned}
$$

where $c_{1}$ is a positive constant which depends on the $C_{b}^{2}(\mathbb{R})$-norm of $f$. It follows that $\left\|\left(\sigma^{\prime} \circ y\right) h\right\|_{X_{\alpha}} \leq c_{2}\|h\|_{X_{\alpha}}\left(1+\|y\|_{X_{\alpha}}\right)$ for every $y, h \in X_{\alpha}$, and some positive constant $c_{2}$, so that $\sigma$ is locally Lipschitz continuous on $X_{\alpha}$. Hence, the assumptions of Theorem 3.1 are satisfied, and problem (3.2) admits a unique mild solution $y$, for any $\psi \in X_{\alpha}$, such that $y(t) \in D\left(A_{p}\right)$ for every $t \in(0, T]$.

Open Access. This article is licensed under a Creative Commons Attribution 4.0 International License, which permits use, sharing, adaptation, distribution and reproduction in any medium or format, as long as you give appropriate credit to the original author(s) and the source, provide a link to the Creative Commons licence, and indicate if changes were made. The images or other third party material in this article are included in the article's Creative Commons licence, unless indicated otherwise in a credit line to the material. If material is not included in the article's Creative Commons licence and your intended use is not permitted by statutory regulation or exceeds the permitted use, you will need to obtain permission directly from the copyright holder. To view a copy of this licence, visit http://creativecommons.org/licenses/ by $/ 4.0 /$.

Publisher's Note Springer Nature remains neutral with regard to jurisdictional claims in published maps and institutional affiliations.

\section{REFERENCES}

[1] P. Cannarsa, G. Da Prato, Stochastic viability for regular closed sets in Hilbert spaces. Rend. Lincei Mat. Appl., 22, 337-346 (2011).

[2] L. Coutin, N. Marie, Invariance for rough differential equations, Stochastic Processes and their Applications 127, 2373-2395 (2017).

[3] A. Deya, M. Gubinelli, S. Tindel, Non-linear rough heat equations, Probab. Theory Related Fields 153, 97-147 (2012).

[4] K.J. Engel, R. Nagel, One-parameter semigroups for linear evolution equations, Springer, New York, NY (2000).

[5] P. Fritz, M Hairer, A Course on Rough Paths With an Introduction to Regularity Structures, Springer Universitext 2014.

[6] M. Gubinelli, Controlling rough paths, J. Funct. Anal., 216, 86-140 (2004).

[7] M. Gubinelli, A panorama of Singular SPDEs, Proceedings of the International Congress of Mathematicians (ICM 2018), World Scientific (2019).

[8] M. Gubinelli, A. Lejay, S. Tindel, Young integrals and SPDEs, Potential Anal. 25, 307-326 (2006).

[9] M. Gubinelli, S. Tindel, Rough evolution equations, Ann. Probab. 38, 1-75 (2010).

[10] A. Lejay, An introduction to rough paths. In: Seminaire de Probabilités XXXVII. Lecture Notes in Mathematics 1832, 1-59. Springer, Berlin Heidelberg New York (2003). 
[11] L. Lorenzi, A. Rhandi, Semigroups of bounded operators and second-order elliptic and parabolic partial differential equations, CRC Press (2021).

[12] A. Lunardi, Analytic semigroups and optimal regularity in parabolic problems, Modern Birkhäuser Classics, Birkhäuser/Springer Basel AG, Basel (1995).

[13] T.J. Lyons, Differential equations driven by rough signals, Rev. Mat. Iberoamericana 14, no. 2, 215-310 (1998).

[14] B. Maslowski, D. Nualart, Evolution equations driven by a fractional Brownian motion. J. Funct. Anal. 202, 277-305 (2003).

[15] L.C. Young, An inequality of the Hölder type, connected with Stieltjes integration, Acta Math. 67, no. 1, 251-282 (1936).

[16] M. Zähle, Integration with respect to fractal functions and stochastic calculus. I. Probab. Theory Related Fields 111, 333-374 (1998).

\author{
Davide Addona and Luca Lorenzi \\ Unity of Mathematics and Computer Science \\ Department of Mathematics, Physics and \\ Computer Sciences \\ University of Parma \\ Parco Area delle Scienze 53/A \\ 43124 Parma \\ Italy \\ E-mail: davide.addona@unipr.it \\ Luca Lorenzi \\ E-mail: luca.lorenzi@unipr.it \\ Gianmario Tessitore \\ Department of Mathematics and Its Applications \\ University of Milano Bicocca \\ Via Roberto Cozzi 55 \\ 20126 Milan \\ Italy \\ E-mail: gianmario.tessitore@unimib.it
}

Accepted: 16 January 2022 